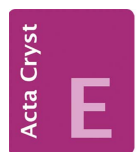

CRYSTALLOGRAPHIC COMMUNICATIONS

ISSN 2056-9890

Received 17 April 2018

Accepted 19 April 2018

Edited by M. Zeller, Purdue University, USA

Keywords: crystal structure; acesulafame; 6methyl-4-oxo-4H-1,2,3-oxathiazin-3-ide 2,2dioxide; strontium; barium.

CCDC references: 1838460; 1838459

Supporting information: this article has supporting information at journals.iucr.org/e

\section{Crystal structure of strontium and barium acesula- fame (6-methyl-4-oxo-4H-1,2,3-oxathiazin-3-ide 2,2-dioxide)}

\author{
Alexander Y. Nazarenko*
}

Chemistry Department, SUNY Buffalo State, 1300 Elmwood Ave, Buffalo, NY 14222, USA. *Correspondence e-mail: nazareay@buffalostate.edu

Both strontium and barium acesulfames, namely poly[aquabis $\left(\mu_{3}-6\right.$-methyl-2,2dioxo-1,2 $\lambda^{6}, 3$-oxathiazin-4-olato)strontium(II)], $\left[\mathrm{Sr}\left(\mathrm{C}_{4} \mathrm{H}_{4} \mathrm{NO}_{4} \mathrm{~S}\right)_{2}\left(\mathrm{H}_{2} \mathrm{O}\right)\right]_{n}$, and the barium(II) analogue, $\left[\mathrm{Ba}\left(\mathrm{C}_{4} \mathrm{H}_{4} \mathrm{NO}_{4} \mathrm{~S}\right)_{2}\left(\mathrm{H}_{2} \mathrm{O}\right)\right]_{n}$, crystallize in nearly identical isotypic forms, with barium-oxygen interatomic distances being longer due to the larger ionic radius of the barium(II) ion. The coordination number of the metal ion is 9; the coordination polyhedra can be described as distorted capped square antiprisms [Johnson solid J10; Johnson (1966). Can. J. Math. 18, 169-200]. The conformation of the acesulafame ions is a distorted envelope with an out-of-plane S atom. Metal and acesulfame ions are assembled into infinitive chains along the [100] axis. These chains are connected via hydrogen bonds into a three-dimensional network.

\section{Chemical context}

Acesulfame is one of the most common sweeteners; usually it is used in the form of a potassium salt. Salts with all alkali metals, ammonium, magnesium, and calcium ions, as well as its protonated molecular form, are also known. The almost identical crystal structures of the strontium and barium salts are reported here.

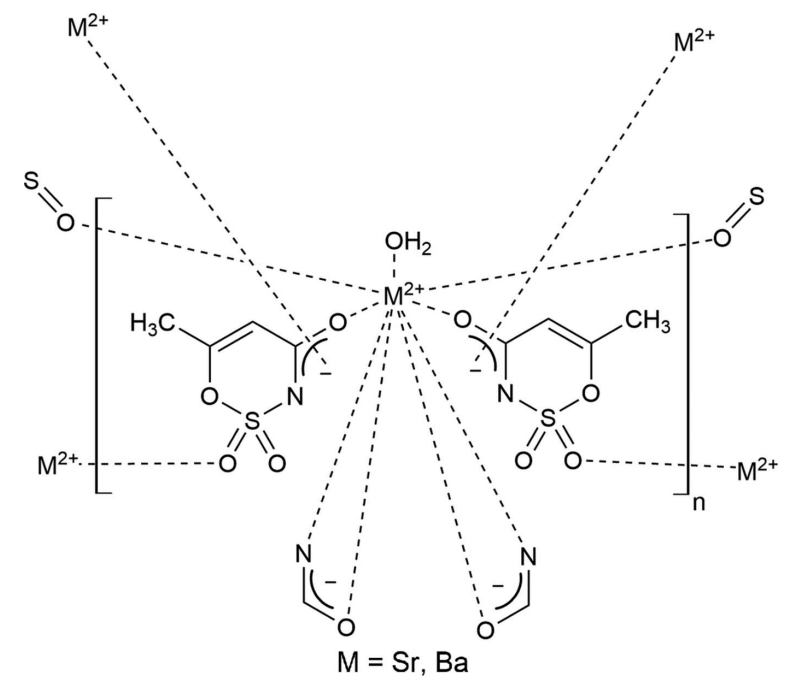

\section{Structural commentary}

Both the strontium and barium compounds crystallize in nearly identical isotypic forms (Fig. 1), with the bariumoxygen interatomic distances being longer due to the larger ionic radius of the barium(II) ion (Tables 1 and 2). Because of 
Table 1

Selected bond lengths $(\AA)$ for Sr.

\begin{tabular}{|c|c|c|c|}
\hline $\mathrm{Sr} 1-\mathrm{O} 1$ & $2.6000(14)$ & $\mathrm{Sr} 1-\mathrm{O} 6^{\mathrm{iii}}$ & $2.8070(13)$ \\
\hline $\mathrm{Sr} 1-\mathrm{O} 2^{\mathrm{i}}$ & 2.9039 (14) & $\mathrm{Sr} 1-\mathrm{O} 9^{\mathrm{iv}}$ & $2.5782(12)$ \\
\hline $\mathrm{Sr} 1-\mathrm{O} 2$ & $2.5026(12)$ & $\mathrm{Sr} 1-\mathrm{N} 1^{\mathrm{i}}$ & $2.7553(15)$ \\
\hline $\mathrm{Sr} 1-\mathrm{O} 5^{\mathrm{ii}}$ & $2.5790(13)$ & $\mathrm{Sr} 1-\mathrm{N} 2^{\mathrm{iii}}$ & $2.7447(14)$ \\
\hline $\mathrm{Sr} 1-\mathrm{O} 6$ & $2.5116(12)$ & & \\
\hline
\end{tabular}

$-x+2,-y+1,-z+1$; (iv) $x-1, y, z$.

Table 2

Selected bond lengths $(\AA)$ for Ba.

\begin{tabular}{llll}
\hline $\mathrm{Ba} 1-\mathrm{O} 1$ & $2.732(2)$ & $\mathrm{Ba} 1-\mathrm{O}^{\mathrm{iii}}$ & $2.9192(18)$ \\
$\mathrm{Ba} 1-\mathrm{O} 2^{\mathrm{i}}$ & $2.9714(19)$ & $\mathrm{Ba} 1-\mathrm{O} 9^{\mathrm{iv}}$ & $2.7478(18)$ \\
$\mathrm{Ba} 1-\mathrm{O} 2$ & $2.6812(17)$ & $\mathrm{Ba} 1-\mathrm{N} 1^{\mathrm{i}}$ & $2.921(2)$ \\
$\mathrm{Ba} 1-\mathrm{O}^{\mathrm{ii}}$ & $2.7560(18)$ & $\mathrm{Ba} 1-\mathrm{N} 2^{\mathrm{iii}}$ & $2.9077(19)$ \\
$\mathrm{Ba} 1-\mathrm{O} 6$ & $2.6788(16)$ & & \\
\hline \multicolumn{5}{l}{ Symmetry codes: } & (i) $-x+1,-y+1,-z+1 ; \quad$ (ii) & $x+1, y, z ; \quad$ (iii) \\
$-x+2,-y+1,-z+1 ;$ (iv) $x-1, y, z$. &
\end{tabular}

the similarity of the structures, representations would be visually identical, and all figures are shown for the strontium salt only. In both cases, the coordination sphere of the metal ion contains a water molecule, two $\mathrm{O}$ atoms from carbonyl groups, two $\mathrm{O}$ atoms from sulfonyl fragments, and two bidentate amide groups coordinated through both their carbonyl group and the adjacent deprotonated $\mathrm{N}$ atom (Fig. 2). The total coordination number is nine. This coordination polyhedron can be described as a distorted capped square antiprism. The corresponding ideal polyhedron is a gyroelongated square pyramid, a Johnson solid J10 (Johnson, 1966). In this case, the base of the polyhedron is not a square but a rectangle formed by two $\mathrm{N}$ and two $\mathrm{O}$ atoms from two bidentate amide groups. Nitrogen-oxygen distances within each of the groups are practically the same: $\mathrm{N} 1^{\mathrm{i}} \cdots \mathrm{O} 2^{\mathrm{i}}=$ 2.232 (2) $\AA$ and $\mathrm{N} 2{ }^{\mathrm{iii}} \ldots \mathrm{O} 6^{\mathrm{iii}}=2.233(2) \AA(\mathrm{Sr})$; and $\mathrm{N} 1^{\mathrm{i}} \ldots \mathrm{O} 2^{\mathrm{i}}$ $=2.233$ (3) $\AA$ and $\mathrm{N} 2{ }^{\mathrm{iii}} \ldots \mathrm{O} 6^{\mathrm{iii}}=2.239$ (2) $\AA$ (Ba). The interatomic separations between these groups are much longer: $\mathrm{N} 1^{\mathrm{i}}{ } \ldots \mathrm{O} 6^{\mathrm{iii}}=3.0609(19)(\mathrm{Sr})$ and $3.174(3) \AA(\mathrm{Ba})$, and $\mathrm{N} 2{ }^{\mathrm{iii}} \cdots \mathrm{O} 2^{\mathrm{i}}=3.0787(18)(\mathrm{Sr})$ and $3.184(3) \AA(\mathrm{Ba})$ [symmetry codes: (i) $-x+1,-y+1,-z+1$; (iii) $-x+2,-y+1,-z+1$.]

Deviations from exact right angles are around $5^{\circ}$ for the $\mathrm{Sr}$ and $1.5^{\circ}$ for the Ba structure. The upper square of the coordination polyhedron is less distorted, with angles very close (mostly within $1^{\circ}$ ) to $90^{\circ}$. The two average planes of the base

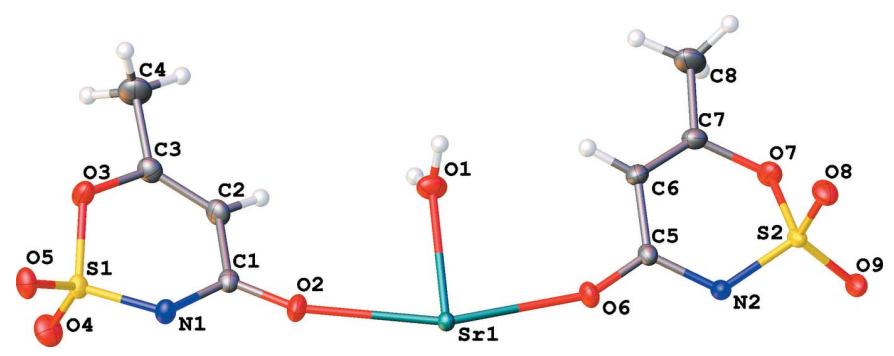

Figure 1

The numbering scheme of strontium acesulfame monohydrate, shown with $50 \%$ probability displacement elipsoids.

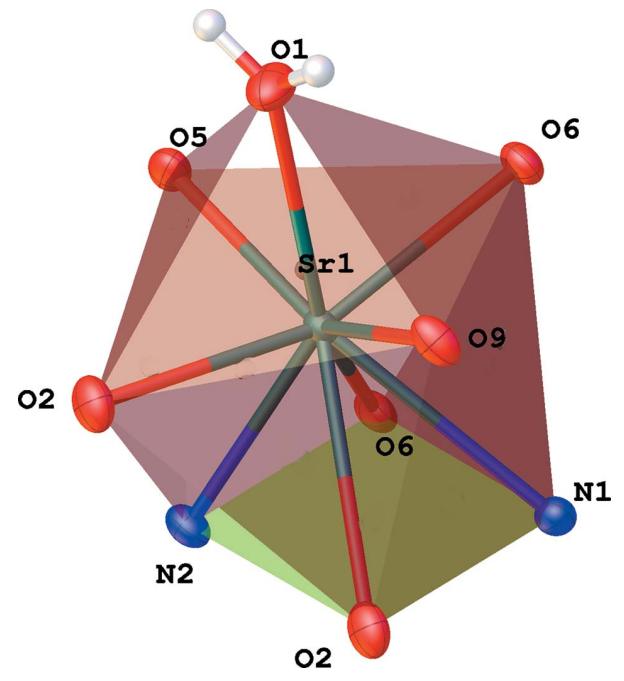

Figure 2

The coordination polyhedron of the Sr metal ion. Symmetry codes for the base rectangle atoms are: $\mathrm{N} 1$ and $\mathrm{O} 2$ at $(-x+1,-y+1,-z+1), \mathrm{N} 2$ and $\mathrm{O} 6$ at $(-x+2,-y+1,-z+1)$. For the upper rectangle: $\mathrm{O} 9$ is at $(x-1, y$, $z)$ and $\mathrm{O} 5$ is at $(x+1, y, z)$.

rectangle and upper square are nearly coplanar, with the angles between them being $1.17(4)(\mathrm{Sr})$ and $0.99(6)^{\circ}(\mathrm{Ba})$. The line between the metal ions and the capping oxygen of the water molecules are nearly perpendicular to these planes: the angles between the upper plane normal and the connecting line are $4.90(4)(\mathrm{Sr})$ and $5.58(6)^{\circ}(\mathrm{Ba})$.

The geometries of the two acesulfame anions in each structure are very similar to each other (Fig. 3), as well as to

Figure 3

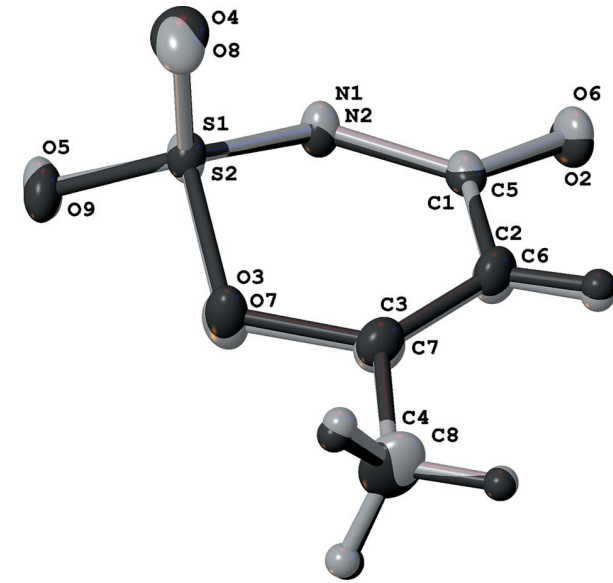

Overlay of the two acesulafame ions in the Sr structure.

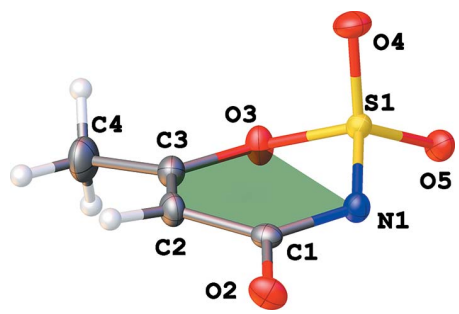

Figure 4

View of the acesulafame anion in the Sr structure. Deviations from the average plane of five atoms in six-membered ring are $0.031(\mathrm{~N} 1),-0.010$ (O3), -0.011 (C3), +0.042 (C2), -0.052 (C1) and 0.5377 (15) A (S1). 
Table 3

Hydrogen-bond geometry $\left(\AA{ }^{\circ}\right)$ for Sr.

\begin{tabular}{lllll}
\hline$D-\mathrm{H} \cdots A$ & $D-\mathrm{H}$ & $\mathrm{H} \cdots A$ & $D \cdots A$ & $D-\mathrm{H} \cdots A$ \\
\hline $\mathrm{O} 1-\mathrm{H} 1 A \cdots \mathrm{O}^{\mathrm{v}}$ & $0.79(3)$ & $2.18(3)$ & $2.899(2)$ & $151(3)$ \\
$\mathrm{O} 1-\mathrm{H} 1 B \cdots 8^{\mathrm{vi}}$ & $0.83(3)$ & $2.22(3)$ & $2.9850(19)$ & $152(3)$ \\
$\mathrm{C} 4-\mathrm{H} 4 B \cdots 5^{\mathrm{v}}$ & 0.98 & 2.37 & $3.273(3)$ & 153 \\
$\mathrm{C} 6-\mathrm{H} 6 \cdots 8^{\mathrm{vi}}$ & 0.95 & 2.47 & $3.318(2)$ & 148 \\
\hline
\end{tabular}

Symmetry codes: (v) $x+\frac{1}{2},-y+\frac{1}{2}, z-\frac{1}{2}$; (vi) $-x+2,-y+1,-z$.

Table 4

Hydrogen-bond geometry $\left(\AA{ }^{\circ}\right)$ for Ba.

\begin{tabular}{lllll}
\hline$D-\mathrm{H} \cdots A$ & $D-\mathrm{H}$ & $\mathrm{H} \cdots A$ & $D \cdots A$ & $D-\mathrm{H} \cdots A$ \\
\hline $\mathrm{O} 1-\mathrm{H} 1 A \cdots \mathrm{O} 4^{\mathrm{v}}$ & $0.74(4)$ & $2.12(4)$ & $2.829(3)$ & $162(4)$ \\
$\mathrm{O} 1-\mathrm{H} 1 B \cdots 8^{\mathrm{vi}}$ & $0.74(4)$ & $2.20(4)$ & $2.889(3)$ & $156(4)$ \\
$\mathrm{C} 4-\mathrm{H} 4 B \cdots 5^{\mathrm{v}}$ & 0.98 & 2.44 & $3.361(4)$ & 155 \\
$\mathrm{C} 6-\mathrm{H} 6 \cdots 8^{\mathrm{vi}}$ & 0.95 & 2.52 & $3.357(3)$ & 147 \\
\hline
\end{tabular}

Symmetry codes: (v) $x+\frac{1}{2},-y+\frac{1}{2}, z-\frac{1}{2}$; (vi) $-x+2,-y+1,-z$.

those of previously reported compounds. The six-membered rings have only one atom with a tetrahedral environment (S). The other five atoms deviate only slightly from their average planes (Fig. 4). This conformation can be described as an envelope, slightly distorted toward a boat.

\section{Supramolecular features}

Each acesulfame anion is connected to three strontium (or barium) ions via a bridging $\mathrm{O}$ atom of the amide carbonyl group, an $\mathrm{N}$ atom of this group, and one of the $\mathrm{O}$ atoms of a sulfonyl group. Each metal ion is directly connected to six acesulfame anions. As a result, metal ions and acesulfame anions form infinite chains along the [100] axis (Fig. 5). The $\mathrm{O}$ atoms of the two sulfonyl groups that are not connected to metal ions form, instead, strong hydrogen bonds with both $\mathrm{H}$ atoms of the water molecule. These hydrogen bonds (Tables 3 and 4) connect each chain to four neighboring parellel chains, thus creating a three-dimensional assembly (Fig. 6). There are

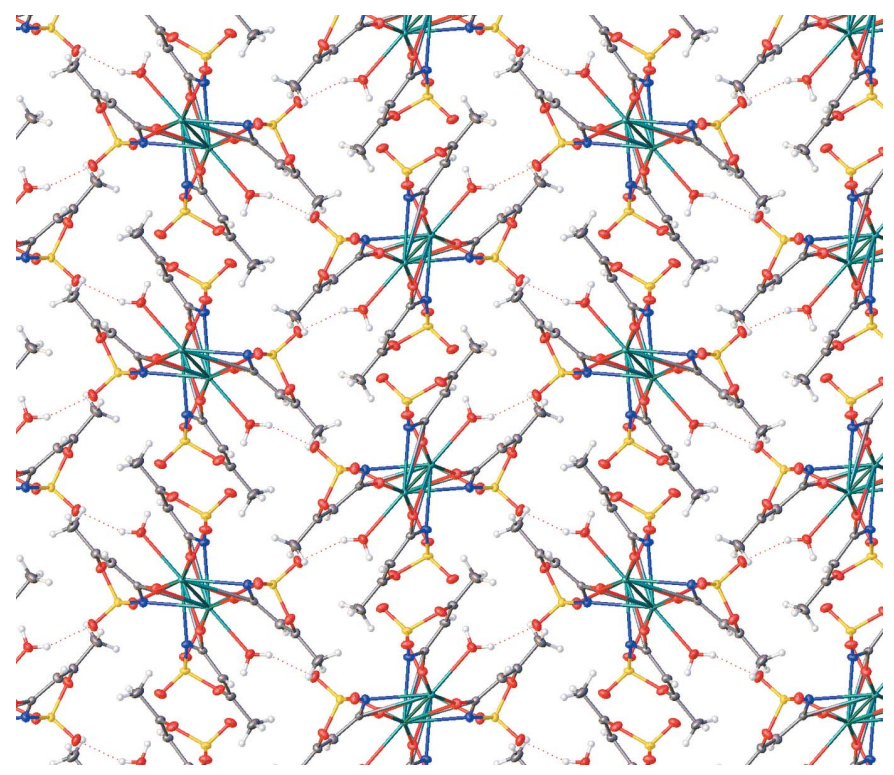

Figure 6

The packing of Sr acesulafame, viewed along the [100] axis.

also two short $\mathrm{C}-\mathrm{H} \cdots \mathrm{O}$ contacts (Tables 3 and 4 ) which may additionally stabilize the crystal structures.

\section{Database survey}

There are over 40 acesulfame structures deposited in the Cambridge Structural Database (CSD; Groom et al., 2016; Version 5.38). Of these structures, alkali metal, ammonium, and thallium salts FEQPUP (Piro et al., 2017), KMTOZD (Paulus, 1975), SUQTOP, SURCIT and SURCOZ (Piro et al., 2015), TOFPEL (Echeverría et al., 2014), OCAHUY (Baran et al., 2015), magnesium salt XAGVAF (Piro et al., 2016), calcium salt EXUCOR (Demirtas et al., 2012), and protonated forms WURMOM and WURMOM01 (Velaga et al., 2010) are closely related to the structures of the title compounds. Several other structures describe coordination compounds

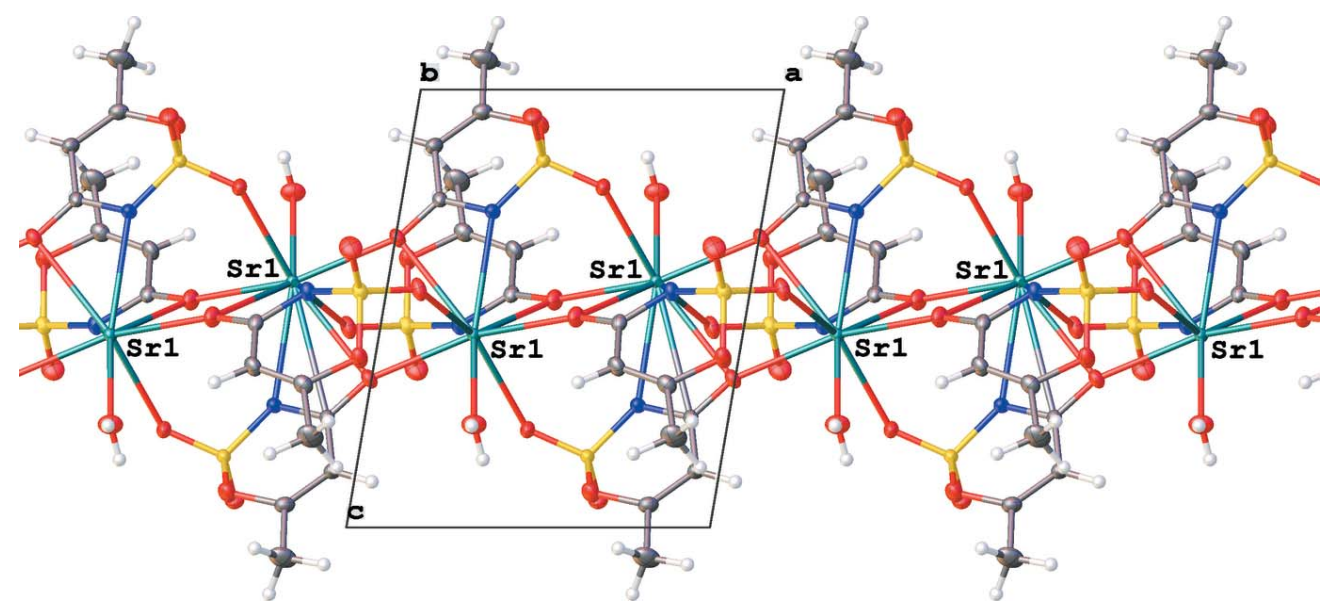

Figure 5

The infinite chain of metal ions and acesulafame anions along the [100] axis. The view is along the [010] vector. 
Table 5

Experimental details.

\begin{tabular}{|c|c|c|}
\hline & Sr complex & Ba complex \\
\hline \multicolumn{3}{|l|}{ Crystal data } \\
\hline Chemical formula & {$\left[\mathrm{Sr}\left(\mathrm{C}_{4} \mathrm{H}_{4} \mathrm{NO}_{4} \mathrm{~S}\right)_{2}\left(\mathrm{H}_{2} \mathrm{O}\right)\right]$} & {$\left[\mathrm{Ba}\left(\mathrm{C}_{4} \mathrm{H}_{4} \mathrm{NO}_{4} \mathrm{~S}\right)_{2}\left(\mathrm{H}_{2} \mathrm{O}\right)\right]$} \\
\hline$M_{\mathrm{r}}$ & 429.92 & 479.64 \\
\hline Crystal system, space group & Monoclinic, $P 2_{1} / n$ & Monoclinic, $P 2_{1} / n$ \\
\hline Temperature $(\mathrm{K})$ & 173 & 173 \\
\hline$a, b, c(\AA)$ & $7.9695(3), 18.5217(8), 9.7310(4)$ & $8.2086(5), 18.8899(11), 9.8999(6)$ \\
\hline$\beta\left({ }^{\circ}\right)$ & $99.651(1)$ & $99.5197(10)$ \\
\hline$V\left(\AA^{6}\right)$ & $1416.05(10)$ & $1513.93(16)$ \\
\hline$Z$ & 4 & 4 \\
\hline Radiation type & Mo $K \alpha$ & Mo $K \alpha$ \\
\hline$\mu\left(\mathrm{mm}^{-1}\right)$ & 4.15 & 2.94 \\
\hline Crystal size $(\mathrm{mm})$ & $0.55 \times 0.13 \times 0.07$ & $0.52 \times 0.21 \times 0.20$ \\
\hline \multicolumn{3}{|l|}{ Data collection } \\
\hline Diffractometer & Bruker PHOTON-100 CMOS & Bruker PHOTON-100 CMOS \\
\hline Absorption correction & Numerical (SADABS; Krause et al., 2015) & Numerical (SADABS; Krause et al., 2015) \\
\hline$T_{\min }, T_{\max }$ & $0.228,0.797$ & $0.309,0.635$ \\
\hline $\begin{array}{l}\text { No. of measured, independent and observed } \\
\quad[I>2 \sigma(I)] \text { reflections }\end{array}$ & $54583,5414,4548$ & $41654,6324,5218$ \\
\hline$R_{\text {int }}$ & 0.043 & 0.048 \\
\hline$(\sin \theta / \lambda)_{\max }\left(\AA^{-1}\right)$ & 0.771 & 0.794 \\
\hline \multicolumn{3}{|l|}{ Refinement } \\
\hline$R\left[F^{2}>2 \sigma\left(F^{2}\right)\right], w R\left(F^{2}\right), S$ & $0.027,0.062,1.04$ & $0.032,0.059,1.09$ \\
\hline No. of reflections & 5414 & 6324 \\
\hline No. of parameters & 209 & 209 \\
\hline $\mathrm{H}$-atom treatment & $\begin{array}{l}\mathrm{H} \text { atoms treated by a mixture of independent } \\
\text { and constrained refinement }\end{array}$ & $\begin{array}{l}\mathrm{H} \text { atoms treated by a mixture of independent } \\
\text { and constrained refinement }\end{array}$ \\
\hline$\Delta \rho_{\max }, \Delta \rho_{\min }\left(\mathrm{e} \AA^{-3}\right)$ & $0.73,-0.46$ & $1.02,-0.61$ \\
\hline
\end{tabular}

Computer programs: APEX2 and SAINT (Bruker, 2013), SHELXT (Sheldrick, 2015a), SHELXL2016 (Sheldrick, 2015b) and OLEX2 (Dolomanov et al., 2009).

with transition-metal ions and various salts of bulky organic cations.

\section{Synthesis and crystallization}

The protonated form of acesulfame, 6-methyl-1,2,3-oxathiazin-4(3H)-one 2,2-dioxide, was synthesized following a published procedure (Velaga et al., 2010). The starting compound, potassium acesulfame, was obtained at a stated purity of $99 \%$ and no attempt was made at further purification. $2 \mathrm{~g}(0.01 \mathrm{~mol})$ of it were dissolved in water $(10 \mathrm{ml})$, acidified with $10 \mathrm{ml}$ of $6 \mathrm{M} \mathrm{HCl}$, and extracted with several portions of methylene chloride $(15 \mathrm{ml}$ each). Evaporation of the methylene chloride extract resulted in crystals of protonated acesulfame (identified by X-ray diffraction; CSD refcode WURMOM). Both strontium oxide (100 mg, $0.001 \mathrm{~mol})$ and barium oxide $(150 \mathrm{mg}, 0.001 \mathrm{~mol})$ were treated with a small amount of water $(\mathrm{ca} 10 \mathrm{ml})$, forming the corresponding hydroxides. Stoichiometric amounts $(0.002 \mathrm{~mol}$, $320 \mathrm{mg}$ ) of 6-methyl-1,2,3-oxathiazin-4(3H)-one 2,2-dioxide were added under gentle heating to neutralize the alkaline solutions. Slow evaporation of the filtrated solutions resulted in colorless crystals (around $100 \mathrm{mg}$ ), some of which were suitable for X-ray investigation. No attempts to optimize the reaction conditions or to recover more material were made. Several crystals were tested; the best results obtained are reported here. FT-IR-ATR, $\mathrm{Sr}$-acesulfame $\left(\mathrm{cm}^{-1}\right)$ : 3620,3550 $\left(\mathrm{H}_{2} \mathrm{O}\right), 1641$ (amide), 1555, $1173\left(\mathrm{SO}_{2}\right), 938$.

\section{Refinement}

Crystal data, data collection and structure refinement details are summarized in Table 5. All $\mathrm{H}$ atoms of water molecules are refined in isotropic approximation. All other $\mathrm{H}$ atoms are refined with riding coordinates; methyl $\mathrm{H}$ atoms are refined as rotating idealized methyl groups and with $U_{\text {iso }}(\mathrm{H})=$ $1.5 U_{\text {iso }}(\mathrm{C})$.

\section{Acknowledgements}

Financial support from the State University of New York for acquisition and maintenance of the X-ray diffractometer is gratefully acknowledged.

\section{References}

Baran, E. J., Parajón-Costa, B. S., Echeverría, G. A. \& Piro, O. E. (2015). Maced. J. Chem. Chem. Eng. 34, 95-100.

Bruker (2013). APEX2 and SAINT. Bruker AXS Inc., Madison, Wisconsin, USA.

Demirtas, G., Dege, N., Icbudak, H., Yurdakul, O. \& Buyukgungor, H. (2012). J. Inorg. Organomet. Polym. 22, 671-679.

Dolomanov, O. V., Bourhis, L. J., Gildea, R. J., Howard, J. A. K. \& Puschmann, H. (2009). J. Appl. Cryst. 42, 339-341.

Echeverría, G. A., Piro, O. E., Parajón-Costa, B. S. \& Baran, E. J. (2014). Z. Naturforsch. Teil B, 69, 737-741.

Groom, C. R., Bruno, I. J., Lightfoot, M. P. \& Ward, S. C. (2016). Acta Cryst. B72, 171-179.

Johnson, N. W. (1966). Can. J. Math. 18, 169-200.

Krause, L., Herbst-Irmer, R., Sheldrick, G. M. \& Stalke, D. (2015). J. Appl. Cryst. 48, 3-10.

Paulus, E. F. (1975). Acta Cryst. B31, 1191-1193. 
Piro, O. E., Echeverría, G. A., Castellano, E. E., Parajón-Costa, B. S. \& Baran, E. J. (2015). Z. Naturforsch. Teil B, 70, 491-496.

Piro, O. E., Echeverría, G. A., Parajón-Costa, B. S. \& Baran, E. J. (2016). Z. Naturforsch. Teil B, 71, 51-56.

Piro, O. E., Echeverría, G. A., Parajón-Costa, B. S. \& Baran, E. J. (2017). J. Chem. Crystallogr. 47, 226-232.
Sheldrick, G. M. (2015a). Acta Cryst. A71, 3-8.

Sheldrick, G. M. (2015b). Acta Cryst. C71, 3-8.

Velaga, S. P., Vangala, V. R., Basavoju, S. \& Bostrom, D. (2010). Chem. Commun. 46, 3562-3564. 


\section{supporting information}

Acta Cryst. (2018). E74, 698-702 [https://doi.org/10.1107/S2056989018006059]

Crystal structure of strontium and barium acesulafame (6-methyl-4oxo-4H-1,2,3-oxathiazin-3-ide 2,2-dioxide)

\section{Alexander Y. Nazarenko}

Computing details

For both structures, data collection: APEX2 (Bruker, 2013); cell refinement: SAINT (Bruker, 2013); data reduction:

SAINT (Bruker, 2013). Program(s) used to solve structure: SHELXT (Sheldrick, 2015a) for Sr. Program(s) used to refine structure: SHELXLL2016 (Sheldrick, 2015b) for Sr; SHELXL97 (Sheldrick, 2008) for Ba. For both structures, molecular graphics: OLEX2 (Dolomanov et al., 2009); software used to prepare material for publication: OLEX2 (Dolomanov et al., 2009).

Poly[aquabis( $\mu_{3}$-6-methyl-2,2-dioxo-1,2 $\lambda^{6}, 3$-oxathiazin-4-olato)strontium(II)] (Sr)

Crystal data

$\left[\mathrm{Sr}\left(\mathrm{C}_{4} \mathrm{H}_{4} \mathrm{NO}_{4} \mathrm{~S}\right)_{2}\left(\mathrm{H}_{2} \mathrm{O}\right)\right]$

$M_{r}=429.92$

Monoclinic, $P 2_{1} / n$

$a=7.9695(3) \AA$

$b=18.5217(8) \AA$

$c=9.7310(4) \AA$

$\beta=99.651(1)^{\circ}$

$V=1416.05(10) \AA^{3}$

$Z=4$

$F(000)=856$

$D_{\mathrm{x}}=2.017 \mathrm{Mg} \mathrm{m}^{-3}$

Mo $K \alpha$ radiation, $\lambda=0.71073 \AA$

Cell parameters from 9082 reflections

$\theta=3.1-33.3^{\circ}$

$\mu=4.15 \mathrm{~mm}^{-1}$

$T=173 \mathrm{~K}$

Needle, colourless

$0.55 \times 0.13 \times 0.07 \mathrm{~mm}$

Data collection

Bruker PHOTON-100 CMOS

diffractometer

$\varphi$ and $\omega$ scans

Absorption correction: numerical

(SADABS; Krause et al., 2015)

$T_{\min }=0.228, T_{\max }=0.797$

54583 measured reflections

\section{Refinement}

Refinement on $F^{2}$

Least-squares matrix: full

$R\left[F^{2}>2 \sigma\left(F^{2}\right)\right]=0.027$

$w R\left(F^{2}\right)=0.062$

$S=1.04$

5414 reflections

209 parameters

0 restraints

Primary atom site location: structure-invariant direct methods
5414 independent reflections

4548 reflections with $I>2 \sigma(I)$

$R_{\text {int }}=0.043$

$\theta_{\max }=33.2^{\circ}, \theta_{\min }=3.1^{\circ}$

$h=-12 \rightarrow 12$

$k=-28 \rightarrow 28$

$l=-14 \rightarrow 14$

Secondary atom site location: difference Fourier map

Hydrogen site location: mixed

$\mathrm{H}$ atoms treated by a mixture of independent and constrained refinement

$w=1 /\left[\sigma^{2}\left(F_{0}^{2}\right)+(0.0247 P)^{2}+1.3759 P\right]$ where $P=\left(F_{\mathrm{o}}^{2}+2 F_{\mathrm{c}}^{2}\right) / 3$

$(\Delta / \sigma)_{\max }=0.001$

$\Delta \rho_{\max }=0.73 \mathrm{e} \AA^{-3}$

$\Delta \rho_{\min }=-0.46 \mathrm{e} \AA^{-3}$ 


\section{Special details}

Geometry. All esds (except the esd in the dihedral angle between two 1.s. planes) are estimated using the full covariance matrix. The cell esds are taken into account individually in the estimation of esds in distances, angles and torsion angles; correlations between esds in cell parameters are only used when they are defined by crystal symmetry. An approximate (isotropic) treatment of cell esds is used for estimating esds involving l.s. planes.

Fractional atomic coordinates and isotropic or equivalent isotropic displacement parameters $\left(\AA^{2}\right)$

\begin{tabular}{lllll}
\hline & $x$ & $y$ & $z$ & $U_{\text {iso }}^{*} / U_{\text {eq }}$ \\
\hline Sr1 & $0.73894(2)$ & $0.46960(2)$ & $0.43922(2)$ & $0.01161(4)$ \\
S1 & $0.07248(5)$ & $0.32528(2)$ & $0.53917(4)$ & $0.01432(7)$ \\
S2 & $1.37055(5)$ & $0.52355(2)$ & $0.16245(4)$ & $0.01457(8)$ \\
O1 & $0.69696(19)$ & $0.37734(8)$ & $0.23495(15)$ & $0.0236(3)$ \\
H1A & $0.703(4)$ & $0.3346(16)$ & $0.232(3)$ & $0.038(8)^{*}$ \\
H1B & $0.656(4)$ & $0.3914(17)$ & $0.155(3)$ & $0.049(9)^{*}$ \\
O2 & $0.46642(16)$ & $0.41317(7)$ & $0.48048(14)$ & $0.0216(3)$ \\
O3 & $0.04339(16)$ & $0.28794(7)$ & $0.38723(14)$ & $0.0207(3)$ \\
O4 & $0.11859(18)$ & $0.26877(7)$ & $0.63821(14)$ & $0.0247(3)$ \\
O5 & $-0.08823(16)$ & $0.35905(7)$ & $0.54351(14)$ & $0.0200(2)$ \\
O6 & $1.00611(16)$ & $0.48946(7)$ & $0.33932(13)$ & $0.0185(2)$ \\
O7 & $1.34454(16)$ & $0.44775(8)$ & $0.07948(14)$ & $0.0218(3)$ \\
O8 & $1.31994(17)$ & $0.57900(8)$ & $0.06234(13)$ & $0.0223(3)$ \\
O9 & $1.54716(15)$ & $0.52276(7)$ & $0.22320(13)$ & $0.0197(2)$ \\
N1 & $0.21644(18)$ & $0.38308(8)$ & $0.53881(15)$ & $0.0159(3)$ \\
N2 & $1.25495(18)$ & $0.51967(8)$ & $0.27867(14)$ & $0.0162(3)$ \\
C1 & $0.3450(2)$ & $0.36951(9)$ & $0.46730(17)$ & $0.0149(3)$ \\
C2 & $0.3318(2)$ & $0.30990(10)$ & $0.36758(18)$ & $0.0191(3)$ \\
H2 & 0.4300 & 0.2956 & 0.3311 & $0.023^{*}$ \\
C3 & $0.1853(2)$ & $0.27495(10)$ & $0.32641(18)$ & $0.0188(3)$ \\
C4 & $0.1419(3)$ & $0.22384(12)$ & $0.2083(2)$ & $0.0310(4)$ \\
H4A & 0.0983 & 0.1789 & 0.2419 & $0.046^{*}$ \\
H4B & 0.2441 & 0.2135 & 0.1680 & $0.046^{*}$ \\
H4C & 0.0548 & 0.2454 & 0.1370 & $0.046^{*}$ \\
C5 & $1.1042(2)$ & $0.48462(9)$ & $0.25133(17)$ & $0.0142(3)$ \\
C6 & $1.0638(2)$ & $0.43825(10)$ & $0.12897(17)$ & $0.0175(3)$ \\
H6 & 0.9511 & 0.4203 & 0.1040 & $0.021^{*}$ \\
C7 & $1.1800(2)$ & $0.42032(10)$ & $0.05129(17)$ & $0.0186(3)$ \\
C8 & $1.1604(3)$ & $0.36965(12)$ & $-0.0686(2)$ & $0.0312(4)$ \\
H8A & 1.1812 & 0.3954 & -0.1521 & $0.047^{*}$ \\
H8B & 1.0446 & 0.3500 & -0.0848 & $0.047^{*}$ \\
H8C & 1.2424 & 0.3301 & -0.0480 & $0.047^{*}$ \\
& & & &
\end{tabular}

Atomic displacement parameters $\left(\AA^{2}\right)$

\begin{tabular}{lllllll}
\hline & $U^{11}$ & $U^{22}$ & $U^{33}$ & $U^{12}$ & $U^{13}$ & $U^{23}$ \\
\hline Sr1 & $0.01046(6)$ & $0.01363(7)$ & $0.01116(6)$ & $-0.00146(5)$ & $0.00300(4)$ & $-0.00080(5)$ \\
S1 & $0.01438(17)$ & $0.01221(16)$ & $0.01734(18)$ & $-0.00014(13)$ & $0.00547(13)$ & $-0.00002(13)$ \\
S2 & $0.01118(16)$ & $0.0224(2)$ & $0.01036(16)$ & $-0.00041(14)$ & $0.00245(12)$ & $-0.00008(14)$
\end{tabular}




\begin{tabular}{lllllll} 
O1 & $0.0324(7)$ & $0.0183(6)$ & $0.0196(6)$ & $-0.0036(5)$ & $0.0034(5)$ & $-0.0044(5)$ \\
O2 & $0.0156(6)$ & $0.0261(7)$ & $0.0230(6)$ & $-0.0074(5)$ & $0.0032(5)$ & $0.0018(5)$ \\
O3 & $0.0161(6)$ & $0.0236(6)$ & $0.0235(6)$ & $-0.0044(5)$ & $0.0061(5)$ & $-0.0086(5)$ \\
O4 & $0.0291(7)$ & $0.0179(6)$ & $0.0278(7)$ & $0.0027(5)$ & $0.0068(5)$ & $0.0079(5)$ \\
O5 & $0.0156(5)$ & $0.0187(6)$ & $0.0280(7)$ & $0.0015(5)$ & $0.0101(5)$ & $0.0016(5)$ \\
O6 & $0.0160(5)$ & $0.0223(6)$ & $0.0189(6)$ & $-0.0016(5)$ & $0.0085(5)$ & $-0.0008(5)$ \\
O7 & $0.0185(6)$ & $0.0283(7)$ & $0.0202(6)$ & $-0.0013(5)$ & $0.0077(5)$ & $-0.0082(5)$ \\
O8 & $0.0196(6)$ & $0.0301(7)$ & $0.0168(6)$ & $0.0013(5)$ & $0.0020(5)$ & $0.0068(5)$ \\
O9 & $0.0113(5)$ & $0.0322(7)$ & $0.0153(5)$ & $-0.0004(5)$ & $0.0016(4)$ & $0.0019(5)$ \\
N1 & $0.0139(6)$ & $0.0156(6)$ & $0.0190(6)$ & $-0.0005(5)$ & $0.0049(5)$ & $-0.0018(5)$ \\
N2 & $0.0132(6)$ & $0.0233(7)$ & $0.0124(6)$ & $-0.0028(5)$ & $0.0029(5)$ & $-0.0021(5)$ \\
C1 & $0.0129(6)$ & $0.0160(7)$ & $0.0155(7)$ & $0.0003(5)$ & $0.0014(5)$ & $0.0042(5)$ \\
C2 & $0.0178(7)$ & $0.0211(8)$ & $0.0201(8)$ & $0.0020(6)$ & $0.0077(6)$ & $-0.0022(6)$ \\
C3 & $0.0213(8)$ & $0.0174(8)$ & $0.0190(8)$ & $0.0012(6)$ & $0.0066(6)$ & $-0.0017(6)$ \\
C4 & $0.0377(11)$ & $0.0292(10)$ & $0.0274(10)$ & $-0.0034(9)$ & $0.0092(8)$ & $-0.0135(8)$ \\
C5 & $0.0120(6)$ & $0.0177(7)$ & $0.0130(7)$ & $0.0006(5)$ & $0.0024(5)$ & $0.0028(5)$ \\
C6 & $0.0162(7)$ & $0.0220(8)$ & $0.0138(7)$ & $-0.0033(6)$ & $0.0008(6)$ & $-0.0008(6)$ \\
C7 & $0.0217(8)$ & $0.0199(8)$ & $0.0137(7)$ & $-0.0022(6)$ & $0.0016(6)$ & $-0.0014(6)$ \\
C8 & $0.0420(12)$ & $0.0308(10)$ & $0.0223(9)$ & $-0.0102(9)$ & $0.0099(8)$ & $-0.0113(8)$ \\
& & & & & & \\
\hline
\end{tabular}

Geometric parameters $\left(\stackrel{\AA}{\circ}{ }^{o}\right)$

\begin{tabular}{llll}
\hline $\mathrm{Sr} 1-\mathrm{Sr} 1^{\mathrm{i}}$ & $4.2816(3)$ & $\mathrm{O} 5-\mathrm{Sr} 1^{\mathrm{iv}}$ & $2.5789(13)$ \\
$\mathrm{Sr} 1-\mathrm{O} 1$ & $2.6000(14)$ & $\mathrm{O} 6-\mathrm{Sr} 1^{\mathrm{i}}$ & $2.8070(13)$ \\
$\mathrm{Sr} 1-\mathrm{O} 2^{\mathrm{ii}}$ & $2.9039(14)$ & $\mathrm{O} 6-\mathrm{C} 5$ & $1.2556(19)$ \\
$\mathrm{Sr} 1-\mathrm{O} 2$ & $2.5026(12)$ & $\mathrm{O} 7-\mathrm{C} 7$ & $1.390(2)$ \\
$\mathrm{Sr} 1-\mathrm{O} 5^{\mathrm{iii}}$ & $2.5790(13)$ & $\mathrm{O} 9-\mathrm{Sr} 1^{\mathrm{iii}}$ & $2.5782(12)$ \\
$\mathrm{Sr} 1-\mathrm{O} 6$ & $2.5116(12)$ & $\mathrm{N} 1-\mathrm{Sr} 1^{\mathrm{ii}}$ & $2.7553(15)$ \\
$\mathrm{Sr} 1-\mathrm{O} 6^{\mathrm{i}}$ & $2.8070(13)$ & $\mathrm{N} 1-\mathrm{C} 1$ & $2.355(2)$ \\
$\mathrm{Sr} 1-\mathrm{O} 9^{\mathrm{iv}}$ & $2.5782(12)$ & $\mathrm{N} 2-\mathrm{Sr} 1^{\mathrm{i}}$ & $1.7448(14)$ \\
$\mathrm{Sr} 1-\mathrm{N} 1^{\mathrm{ii}}$ & $2.7553(15)$ & $\mathrm{N} 2-\mathrm{C} 5$ & $3.2186(17)$ \\
$\mathrm{Sr} 1-\mathrm{N} 2^{\mathrm{i}}$ & $2.7447(14)$ & $\mathrm{C} 1-\mathrm{Sr} 1^{\mathrm{ii}}$ & $1.462(2)$ \\
$\mathrm{Sr} 1-\mathrm{C} 1^{\mathrm{ii}}$ & $3.2186(17)$ & $\mathrm{C} 1-\mathrm{C} 2$ & 0.9500 \\
$\mathrm{Sr} 1-\mathrm{C} 5^{\mathrm{i}}$ & $3.1761(16)$ & $\mathrm{C} 2-\mathrm{H} 2$ & $1.336(2)$ \\
$\mathrm{S} 1-\mathrm{O} 3$ & $1.6134(13)$ & $\mathrm{C} 2-\mathrm{C} 3$ & $1.484(3)$ \\
$\mathrm{S} 1-\mathrm{O} 4$ & $1.4285(13)$ & $\mathrm{C} 3-\mathrm{C} 4$ & 0.9800 \\
$\mathrm{~S} 1-\mathrm{O} 5$ & $1.4323(13)$ & $\mathrm{C} 4-\mathrm{H} 4 \mathrm{~A}$ & 0.9800 \\
$\mathrm{~S} 1-\mathrm{N} 1$ & $1.5697(15)$ & $\mathrm{C} 4-\mathrm{H} 4 \mathrm{~B}$ & 0.9800 \\
$\mathrm{~S} 2-\mathrm{O} 7$ & $1.6156(14)$ & $\mathrm{C} 4-\mathrm{H} 4 \mathrm{C}$ & $3.1762(16)$ \\
$\mathrm{S} 2-\mathrm{O} 8$ & $1.4264(13)$ & $\mathrm{C} 5-\mathrm{Sr} 1^{\mathrm{i}}$ & $1.460(2)$ \\
$\mathrm{S} 2-\mathrm{O} 9$ & $1.4324(13)$ & $\mathrm{C} 5-\mathrm{C} 6$ & 0.9500 \\
$\mathrm{~S} 2-\mathrm{N} 2$ & $1.5755(14)$ & $\mathrm{C} 6-\mathrm{H} 6$ & $1.332(2)$ \\
$\mathrm{O} 1-\mathrm{H} 1 \mathrm{~A}$ & $0.79(3)$ & $\mathrm{C} 6-\mathrm{C} 7$ & $1.485(3)$ \\
$\mathrm{O} 1-\mathrm{H} 1 \mathrm{~B}$ & $0.84(3)$ & $\mathrm{C} 7-\mathrm{C} 8$ & 0.9800 \\
$\mathrm{O} 2-\mathrm{Sr} 1^{\mathrm{ii}}$ & $2.9040(14)$ & $\mathrm{C} 8-\mathrm{H} 8 \mathrm{~A}$ & 0.9800 \\
$\mathrm{O} 2-\mathrm{C} 1$ & $1.251(2)$ & $\mathrm{C} 8-\mathrm{H} 8 \mathrm{~B}$ & 0.9800 \\
$\mathrm{O} 3-\mathrm{C} 3$ & $1.383(2)$ & $\mathrm{C} 8-\mathrm{H} 8 \mathrm{C}$ &
\end{tabular}




$$
\begin{aligned}
& \mathrm{O} 1-\mathrm{Sr} 1-\mathrm{Sr} 1^{\mathrm{i}} \\
& \mathrm{O} 1-\mathrm{Sr} 1-\mathrm{O} 2^{\mathrm{ii}} \\
& \mathrm{O} 1-\mathrm{Sr} 1-\mathrm{O}^{\mathrm{i}} \\
& \mathrm{O} 1-\mathrm{Sr} 1-\mathrm{N} 1^{\text {ii }} \\
& \mathrm{O} 1-\mathrm{Sr} 1-\mathrm{N} 2^{\mathrm{i}} \\
& \mathrm{O} 1-\mathrm{Sr} 1-\mathrm{C}^{1 i} \\
& \mathrm{O} 1-\mathrm{Sr} 1-\mathrm{C} 5^{\mathrm{i}} \\
& \mathrm{O} 2-\mathrm{Sr} 1-\mathrm{Sr}^{\mathrm{i}} \\
& \mathrm{O} 2^{\mathrm{ii}}-\mathrm{Sr} 1-\mathrm{Sr}^{\mathrm{i}} \\
& \mathrm{O} 2-\mathrm{Sr} 1-\mathrm{O} 1 \\
& \mathrm{O} 2-\mathrm{Sr} 1-\mathrm{O} 2^{\mathrm{ii}} \\
& \mathrm{O} 2-\mathrm{Sr} 1-\mathrm{O} 5^{\mathrm{iii}} \\
& \mathrm{O} 2-\mathrm{Sr} 1-\mathrm{O} 6 \\
& \mathrm{O} 2-\mathrm{Sr} 1-\mathrm{O}^{\mathrm{i}} \\
& \mathrm{O} 2-\mathrm{Sr} 1-\mathrm{O}^{\text {iv }} \\
& \mathrm{O} 2-\mathrm{Sr} 1-\mathrm{N} 1^{i i} \\
& \mathrm{O} 2-\mathrm{Sr} 1-\mathrm{N} 2^{\mathrm{i}} \\
& \mathrm{O} 2^{\mathrm{ii}}-\mathrm{Sr} 1-\mathrm{C}^{\mathrm{ii}} \\
& \mathrm{O} 2-\mathrm{Sr} 1-\mathrm{Cl}^{\mathrm{ii}} \\
& \mathrm{O} 2^{\mathrm{ii}}-\mathrm{Sr} 1-\mathrm{C} 5^{\mathrm{i}} \\
& \mathrm{O} 2-\mathrm{Sr} 1-\mathrm{C} 5^{\mathrm{i}}
\end{aligned}
$$

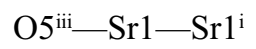

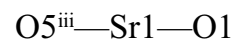

$$
\begin{aligned}
& \mathrm{O} 5^{\mathrm{iii}}-\mathrm{Sr} 1-\mathrm{O} 2^{\mathrm{ii}}
\end{aligned}
$$

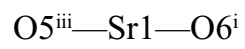

$$
\begin{aligned}
& \mathrm{O} 5^{\mathrm{iii}-\mathrm{S}} 1-\mathrm{N} 1^{\mathrm{ii}}
\end{aligned}
$$

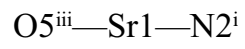

$$
\begin{aligned}
& \mathrm{O}{ }^{i i i}-\mathrm{Sr} 1-\mathrm{C}^{\mathrm{ii}}
\end{aligned}
$$

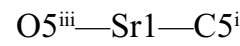

$$
\begin{aligned}
& \mathrm{O} 6{ }^{\mathrm{i}}-\mathrm{Sr} 1-\mathrm{Sr} 1^{\mathrm{i}} \\
& \text { O6- } \mathrm{Sr} 1-\mathrm{Sr}^{\mathrm{i}} \\
& \text { O6- } \mathrm{Sr} 1-\mathrm{O} 1 \\
& \mathrm{O} 6-\mathrm{Sr} 1-\mathrm{O} 2^{\mathrm{ii}} \\
& \mathrm{O} 6-\mathrm{Sr} 1-\mathrm{O}^{\mathrm{ii}} \\
& \text { O6- } \mathrm{Sr} 1-\mathrm{O} 5^{\mathrm{iii}} \\
& \text { O6- } \mathrm{Sr} 1-\mathrm{O}^{\mathrm{i}} \\
& \text { O6- } \mathrm{Sr} 1-\mathrm{O}^{\text {iv }} \\
& \text { O6- } \mathrm{Sr} 1-\mathrm{N} 1^{1 i} \\
& \mathrm{O} 6-\mathrm{Sr} 1-\mathrm{N} 2^{\mathrm{i}} \\
& \mathrm{O} 6{ }^{\mathrm{i}}-\mathrm{Sr} 1-\mathrm{C}^{\mathrm{ii}} \\
& \text { O6- } \mathrm{Sr} 1-\mathrm{Cl}^{\mathrm{ii}} \\
& \mathrm{O} 6-\mathrm{Sr} 1-\mathrm{C} 5^{\mathrm{i}} \\
& \mathrm{O} 6{ }^{\mathrm{i}}-\mathrm{Sr} 1-\mathrm{C} 5^{\mathrm{i}} \\
& \mathrm{O} 9^{\mathrm{iv}}-\mathrm{Sr} 1-\mathrm{Sr} 1^{\mathrm{i}} \\
& \mathrm{O} 9^{\mathrm{iv}}-\mathrm{Sr} 1-\mathrm{O} 1 \\
& \mathrm{O}^{\mathrm{iv}}-\mathrm{Sr} 1-\mathrm{O}^{2 \mathrm{ii}} \\
& \mathrm{O} 9^{\mathrm{iv}}-\mathrm{Sr} 1-\mathrm{O} 5^{\mathrm{iii}} \\
& \mathrm{O}^{\mathrm{iv}}-\mathrm{Sr} 1-\mathrm{O}^{\mathrm{i}}
\end{aligned}
$$

$112.25(3)$

$133.71(4)$

$138.44(4)$

$135.12(5)$

$142.25(5)$

$144.92(5)$

$151.65(5)$

$153.49(3)$

$107.12(3)$

$81.04(5)$

\begin{tabular}{|c|c|}
\hline $\mathrm{O} 4-\mathrm{S} 1-\mathrm{O} 5$ & $115.48(8)$ \\
\hline $\mathrm{O} 4-\mathrm{S} 1-\mathrm{N} 1$ & $113.32(8)$ \\
\hline $\mathrm{O} 5-\mathrm{S} 1-\mathrm{O} 3$ & $102.85(7)$ \\
\hline $\mathrm{O} 5-\mathrm{S} 1-\mathrm{N} 1$ & $111.08(8)$ \\
\hline $\mathrm{N} 1-\mathrm{S} 1-\mathrm{O} 3$ & $106.45(7)$ \\
\hline $\mathrm{O} 8-\mathrm{S} 2-\mathrm{O} 7$ & $106.65(8)$ \\
\hline $\mathrm{O} 8-\mathrm{S} 2-\mathrm{O} 9$ & $115.79(8)$ \\
\hline $\mathrm{O} 8-\mathrm{S} 2-\mathrm{N} 2$ & $113.00(8)$ \\
\hline $\mathrm{O} 9-\mathrm{S} 2-\mathrm{O} 7$ & $103.43(8)$ \\
\hline $\mathrm{O} 9-\mathrm{S} 2-\mathrm{N} 2$ & $110.83(8)$ \\
\hline $\mathrm{N} 2-\mathrm{S} 2-\mathrm{O} 7$ & $106.12(7)$ \\
\hline $\mathrm{Sr} 1-\mathrm{O} 1-\mathrm{H} 1 \mathrm{~A}$ & $133(2)$ \\
\hline $\mathrm{Sr} 1-\mathrm{O} 1-\mathrm{H} 1 \mathrm{~B}$ & $119(2)$ \\
\hline $\mathrm{H} 1 \mathrm{~A}-\mathrm{O} 1-\mathrm{H} 1 \mathrm{~B}$ & $107(3)$ \\
\hline $\mathrm{Sr} 1-\mathrm{O} 2-\mathrm{Sr} 1^{\mathrm{ii}}$ & $105.92(5)$ \\
\hline $\mathrm{C} 1-\mathrm{O} 2-\mathrm{Sr} 1$ & $158.55(12)$ \\
\hline $\mathrm{C} 1-\mathrm{O} 2-\mathrm{Sr} 1^{\mathrm{ii}}$ & $92.84(10)$ \\
\hline $\mathrm{C} 3-\mathrm{O} 3-\mathrm{S} 1$ & $117.69(11)$ \\
\hline $\mathrm{S} 1-\mathrm{O} 5-\mathrm{Sr} 1^{\mathrm{iv}}$ & $139.14(7)$ \\
\hline $\mathrm{Sr} 1-\mathrm{O} 6-\mathrm{Sr} 1^{\mathrm{i}}$ & $107.09(4)$ \\
\hline $\mathrm{C} 5-\mathrm{O} 6-\mathrm{Sr}^{\mathrm{i}}$ & $95.14(10)$ \\
\hline $\mathrm{C} 5-\mathrm{O} 6-\mathrm{Sr} 1$ & $156.71(12)$ \\
\hline $\mathrm{C} 7-\mathrm{O} 7-\mathrm{S} 2$ & $117.00(11)$ \\
\hline $\mathrm{S} 2-\mathrm{O} 9-\mathrm{Sr} 1^{\mathrm{iii}}$ & $138.38(8)$ \\
\hline $\mathrm{S} 1-\mathrm{N} 1-\mathrm{Sr} 1^{\mathrm{ii}}$ & $139.48(8)$ \\
\hline $\mathrm{C} 1-\mathrm{N} 1-\mathrm{Sr} 1^{\mathrm{ii}}$ & $97.17(10)$ \\
\hline $\mathrm{C} 1-\mathrm{N} 1-\mathrm{S} 1$ & $119.36(12)$ \\
\hline $\mathrm{S} 2-\mathrm{N} 2-\mathrm{Sr} 1^{\mathrm{i}}$ & $143.15(8)$ \\
\hline $\mathrm{C} 5-\mathrm{N} 2-\mathrm{Sr} 1^{\mathrm{i}}$ & $95.61(9)$ \\
\hline $\mathrm{C} 5-\mathrm{N} 2-\mathrm{S} 2$ & $119.20(12)$ \\
\hline $\mathrm{O} 2-\mathrm{C} 1-\mathrm{Sr}^{\mathrm{ii}}$ & $64.31(9)$ \\
\hline $\mathrm{O} 2-\mathrm{C} 1-\mathrm{N} 1$ & $117.77(16)$ \\
\hline $\mathrm{O} 2-\mathrm{C} 1-\mathrm{C} 2$ & $121.63(15)$ \\
\hline $\mathrm{N} 1-\mathrm{C} 1-\mathrm{Sr} 1^{\mathrm{ii}}$ & $58.14(9)$ \\
\hline $\mathrm{N} 1-\mathrm{C} 1-\mathrm{C} 2$ & $120.30(15)$ \\
\hline $\mathrm{C} 2-\mathrm{C} 1-\mathrm{Sr} 1^{\mathrm{ii}}$ & $153.07(11)$ \\
\hline $\mathrm{C} 1-\mathrm{C} 2-\mathrm{H} 2$ & 119.1 \\
\hline $\mathrm{C} 3-\mathrm{C} 2-\mathrm{C} 1$ & $121.80(15)$ \\
\hline $\mathrm{C} 3-\mathrm{C} 2-\mathrm{H} 2$ & 119.1 \\
\hline $\mathrm{O} 3-\mathrm{C} 3-\mathrm{C} 4$ & $109.98(16)$ \\
\hline $\mathrm{C} 2-\mathrm{C} 3-\mathrm{O} 3$ & $121.56(16)$ \\
\hline $\mathrm{C} 2-\mathrm{C} 3-\mathrm{C} 4$ & $128.27(17)$ \\
\hline $\mathrm{C} 3-\mathrm{C} 4-\mathrm{H} 4 \mathrm{~A}$ & 109.5 \\
\hline $\mathrm{C} 3-\mathrm{C} 4-\mathrm{H} 4 \mathrm{~B}$ & 109.5 \\
\hline $\mathrm{C} 3-\mathrm{C} 4-\mathrm{H} 4 \mathrm{C}$ & 109.5 \\
\hline $\mathrm{H} 4 \mathrm{~A}-\mathrm{C} 4-\mathrm{H} 4 \mathrm{~B}$ & 109.5 \\
\hline $\mathrm{H} 4 \mathrm{~A}-\mathrm{C} 4-\mathrm{H} 4 \mathrm{C}$ & 109.5 \\
\hline $\mathrm{H} 4 \mathrm{~B}-\mathrm{C} 4-\mathrm{H} 4 \mathrm{C}$ & 109.5 \\
\hline
\end{tabular}

$74.08(5)$

91.48 (4)

159.74 (4)

$121.71(4)$

$83.02(4)$

120.35 (4)

74.94 (4)

22.85 (4)

96.57 (4)

$72.26(4)$

$100.00(4)$

$70.73(3)$

$76.75(5)$

140.87 (4)

$69.04(4)$

$134.61(4)$

75.24 (4)

$138.28(4)$

$74.90(4)$

$34.10(2)$

$38.80(3)$

$79.00(4)$

$87.68(4)$

$123.18(4)$

$80.62(4)$

$72.91(4)$

93.08 (4)

77.19 (4)

120.16 (4)

71.99 (4)

101.74 (4)

95.93 (4)

23.19 (4)

122.73 (3)

$69.20(5)$

69.51 (4)

145.95 (4)

140.95 (4) 


\begin{tabular}{|c|c|c|c|}
\hline $\mathrm{O} 9^{\mathrm{iv}}-\mathrm{Sr} 1-\mathrm{N} 1^{\mathrm{ii}}$ & $74.71(4)$ & $\mathrm{O} 6-\mathrm{C} 5-\mathrm{Sr}^{\mathrm{i}}$ & $61.67(9)$ \\
\hline $\mathrm{O} 9^{\mathrm{iv}}-\mathrm{Sr} 1-\mathrm{N} 2^{\mathrm{i}}$ & $134.15(4)$ & $\mathrm{O} 6-\mathrm{C} 5-\mathrm{N} 2$ & $117.76(15)$ \\
\hline $\mathrm{O} 9^{\mathrm{iv}}-\mathrm{Sr} 1-\mathrm{C} 1^{\mathrm{ii}}$ & $75.76(4)$ & $\mathrm{O} 6-\mathrm{C} 5-\mathrm{C} 6$ & $121.39(15)$ \\
\hline $\mathrm{O} 9^{\mathrm{iv}}-\mathrm{Sr} 1-\mathrm{C} 5^{\mathrm{i}}$ & $139.15(4)$ & $\mathrm{N} 2-\mathrm{C} 5-\mathrm{Sr}^{\mathrm{i}}$ & $59.32(8)$ \\
\hline $\mathrm{N} 1^{\mathrm{ii}}-\mathrm{Sr} 1-\mathrm{Sr} 1^{\mathrm{i}}$ & $67.12(3)$ & $\mathrm{N} 2-\mathrm{C} 5-\mathrm{C} 6$ & $120.67(14)$ \\
\hline $\mathrm{N} 1^{\mathrm{ii}}-\mathrm{Sr} 1-\mathrm{O} 2^{\mathrm{ii}}$ & $46.36(4)$ & $\mathrm{C} 6-\mathrm{C} 5-\mathrm{Sr}^{\mathrm{i}}$ & $158.19(11)$ \\
\hline $\mathrm{N} 1^{\mathrm{ii}}-\mathrm{Sr} 1-\mathrm{O}^{\mathrm{i}}$ & $66.77(4)$ & $\mathrm{C} 5-\mathrm{C} 6-\mathrm{H} 6$ & 119.0 \\
\hline $\mathrm{N} 1^{\mathrm{ii}}-\mathrm{Sr} 1-\mathrm{C} 1^{\mathrm{ii}}$ & $24.69(4)$ & $\mathrm{C} 7-\mathrm{C} 6-\mathrm{C} 5$ & $122.03(16)$ \\
\hline $\mathrm{N} 1^{\mathrm{ii}}-\mathrm{Sr} 1-\mathrm{C} 5^{\mathrm{i}}$ & $68.71(4)$ & $\mathrm{C} 7-\mathrm{C} 6-\mathrm{H} 6$ & 119.0 \\
\hline $\mathrm{N} 2^{\mathrm{i}}-\mathrm{Sr} 1-\mathrm{Sr} 1^{\mathrm{i}}$ & $81.44(3)$ & $\mathrm{O} 7-\mathrm{C} 7-\mathrm{C} 8$ & $110.95(16)$ \\
\hline $\mathrm{N} 2^{\mathrm{i}}-\mathrm{Sr} 1-\mathrm{O} 2^{\mathrm{ii}}$ & $65.98(4)$ & $\mathrm{C} 6-\mathrm{C} 7-\mathrm{O} 7$ & $121.31(15)$ \\
\hline $\mathrm{N} 2^{\mathrm{i}}-\mathrm{Sr} 1-\mathrm{O} 6^{\mathrm{i}}$ & $47.42(4)$ & $\mathrm{C} 6-\mathrm{C} 7-\mathrm{C} 8$ & $127.71(17)$ \\
\hline $\mathrm{N} 2{ }^{\mathrm{i}}-\mathrm{Sr} 1-\mathrm{N} 1^{\mathrm{ii}}$ & $82.54(4)$ & $\mathrm{C} 7-\mathrm{C} 8-\mathrm{H} 8 \mathrm{~A}$ & 109.5 \\
\hline $\mathrm{N} 2{ }^{\mathrm{i}}-\mathrm{Sr} 1-\mathrm{C} 1^{\mathrm{ii}}$ & $67.66(4)$ & $\mathrm{C} 7-\mathrm{C} 8-\mathrm{H} 8 \mathrm{~B}$ & 109.5 \\
\hline $\mathrm{N} 2^{\mathrm{i}}-\mathrm{Sr} 1-\mathrm{C} 5^{\mathrm{i}}$ & $25.07(4)$ & $\mathrm{C} 7-\mathrm{C} 8-\mathrm{H} 8 \mathrm{C}$ & 109.5 \\
\hline $\mathrm{C} 1^{\mathrm{ii}}-\mathrm{Sr} 1-\mathrm{Sr} 1^{\mathrm{i}}$ & $85.22(3)$ & $\mathrm{H} 8 \mathrm{~A}-\mathrm{C} 8-\mathrm{H} 8 \mathrm{~B}$ & 109.5 \\
\hline $\mathrm{C} 5^{\mathrm{i}}-\mathrm{Sr} 1-\mathrm{Sr} 1^{\mathrm{i}}$ & $57.18(3)$ & $\mathrm{H} 8 \mathrm{~A}-\mathrm{C} 8-\mathrm{H} 8 \mathrm{C}$ & 109.5 \\
\hline $\mathrm{C} 5^{\mathrm{i}}-\mathrm{Sr} 1-\mathrm{C} 1^{\mathrm{ii}}$ & $63.40(4)$ & $\mathrm{H} 8 \mathrm{~B}-\mathrm{C} 8-\mathrm{H} 8 \mathrm{C}$ & 109.5 \\
\hline $\mathrm{O} 4-\mathrm{S} 1-\mathrm{O} 3$ & $106.58(8)$ & & \\
\hline $\mathrm{Sr} 1-\mathrm{O} 2-\mathrm{C} 1-\mathrm{Sr} 1^{\mathrm{ii}}$ & $-151.3(3)$ & $\mathrm{O} 3-\mathrm{S} 1-\mathrm{N} 1-\mathrm{C} 1$ & $-35.06(15)$ \\
\hline $\mathrm{Sr} 1-\mathrm{O} 2-\mathrm{C} 1-\mathrm{N} 1$ & $-175.1(2)$ & $\mathrm{O} 4-\mathrm{S} 1-\mathrm{O} 3-\mathrm{C} 3$ & $-82.71(14)$ \\
\hline $\mathrm{Sr} 1 \mathrm{ii}-\mathrm{O} 2-\mathrm{C} 1-\mathrm{N} 1$ & $-23.76(15)$ & $\mathrm{O} 4-\mathrm{S} 1-\mathrm{O} 5-\mathrm{Sr}^{1 \mathrm{iv}}$ & $170.15(11)$ \\
\hline $\mathrm{Sr} 1-\mathrm{O} 2-\mathrm{C} 1-\mathrm{C} 2$ & $-1.4(4)$ & $\mathrm{O} 4-\mathrm{S} 1-\mathrm{N} 1-\mathrm{Sr} 1^{\mathrm{ii}}$ & $-126.76(12)$ \\
\hline $\mathrm{Sr} 1^{\mathrm{ii}}-\mathrm{O} 2-\mathrm{C} 1-\mathrm{C} 2$ & $149.95(14)$ & $\mathrm{O} 4-\mathrm{S} 1-\mathrm{N} 1-\mathrm{C} 1$ & $81.76(15)$ \\
\hline $\mathrm{Sr} 1-\mathrm{O} 6-\mathrm{C} 5-\mathrm{Sr} 1^{\mathrm{i}}$ & $-162.9(3)$ & $\mathrm{O} 5-\mathrm{S} 1-\mathrm{O} 3-\mathrm{C} 3$ & $155.40(13)$ \\
\hline $\mathrm{Sr} 1 \mathrm{i}-\mathrm{O} 6-\mathrm{C} 5-\mathrm{N} 2$ & $-20.16(16)$ & $\mathrm{O} 5-\mathrm{S} 1-\mathrm{N} 1-\mathrm{Sr}^{1 \mathrm{ii}}$ & $5.17(15)$ \\
\hline $\mathrm{Sr} 1-\mathrm{O} 6-\mathrm{C} 5-\mathrm{N} 2$ & $177.0(2)$ & $\mathrm{O} 5-\mathrm{S} 1-\mathrm{N} 1-\mathrm{C} 1$ & $-146.30(13)$ \\
\hline $\mathrm{Sr} 1-\mathrm{O} 6-\mathrm{C} 5-\mathrm{C} 6$ & $-7.8(4)$ & $\mathrm{O} 6-\mathrm{C} 5-\mathrm{C} 6-\mathrm{C} 7$ & $-165.34(17)$ \\
\hline $\mathrm{Sr} 1^{\mathrm{i}}-\mathrm{O} 6-\mathrm{C} 5-\mathrm{C} 6$ & $155.04(14)$ & $\mathrm{O} 7-\mathrm{S} 2-\mathrm{O} 9-\mathrm{Sr}{ }^{\mathrm{iii}}$ & $-82.06(12)$ \\
\hline $\mathrm{Sr} 1^{\mathrm{ii}}-\mathrm{N} 1-\mathrm{C} 1-\mathrm{O} 2$ & $25.31(16)$ & $\mathrm{O} 7-\mathrm{S} 2-\mathrm{N} 2-\mathrm{Sr} 1^{\mathrm{i}}$ & $123.73(13)$ \\
\hline $\mathrm{Sr} 1^{\mathrm{ii}}-\mathrm{N} 1-\mathrm{C} 1-\mathrm{C} 2$ & $-148.50(13)$ & $\mathrm{O} 7-\mathrm{S} 2-\mathrm{N} 2-\mathrm{C} 5$ & $-35.08(15)$ \\
\hline $\mathrm{Sr} 1^{\mathrm{i}}-\mathrm{N} 2-\mathrm{C} 5-\mathrm{O} 6$ & $20.65(16)$ & $\mathrm{O} 8-\mathrm{S} 2-\mathrm{O} 7-\mathrm{C} 7$ & $-79.80(14)$ \\
\hline $\mathrm{Sr} 1^{\mathrm{i}}-\mathrm{N} 2-\mathrm{C} 5-\mathrm{C} 6$ & $-154.58(13)$ & $\mathrm{O} 8-\mathrm{S} 2-\mathrm{O} 9-\mathrm{Sr}{ }^{\mathrm{iii}}$ & $161.68(10)$ \\
\hline $\mathrm{Sr} 1^{\mathrm{ii}}-\mathrm{C} 1-\mathrm{C} 2-\mathrm{C} 3$ & $-67.2(3)$ & $\mathrm{O} 8-\mathrm{S} 2-\mathrm{N} 2-\mathrm{Sr} 1^{\mathrm{i}}$ & $-119.74(13)$ \\
\hline 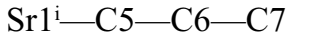 & $-73.7(4)$ & $\mathrm{O} 8-\mathrm{S} 2-\mathrm{N} 2-\mathrm{C} 5$ & $81.44(15)$ \\
\hline $\mathrm{S} 1-\mathrm{O} 3-\mathrm{C} 3-\mathrm{C} 2$ & $-19.8(2)$ & $\mathrm{O} 9-\mathrm{S} 2-\mathrm{O} 7-\mathrm{C} 7$ & $157.64(12)$ \\
\hline $\mathrm{S} 1-\mathrm{O} 3-\mathrm{C} 3-\mathrm{C} 4$ & $164.84(13)$ & $\mathrm{O} 9-\mathrm{S} 2-\mathrm{N} 2-\mathrm{Sr} 1^{\mathrm{i}}$ & $12.10(16)$ \\
\hline $\mathrm{S} 1-\mathrm{N} 1-\mathrm{C} 1-\mathrm{Sr} 1^{\mathrm{ii}}$ & $161.78(14)$ & $\mathrm{O} 9-\mathrm{S} 2-\mathrm{N} 2-\mathrm{C} 5$ & $-146.71(13)$ \\
\hline $\mathrm{S} 1-\mathrm{N} 1-\mathrm{C} 1-\mathrm{O} 2$ & $-172.91(13)$ & $\mathrm{N} 1-\mathrm{S} 1-\mathrm{O} 3-\mathrm{C} 3$ & $38.52(15)$ \\
\hline $\mathrm{S} 1-\mathrm{N} 1-\mathrm{C} 1-\mathrm{C} 2$ & $13.3(2)$ & $\mathrm{N} 1-\mathrm{S} 1-\mathrm{O} 5-\mathrm{Sr} 1^{\mathrm{iv}}$ & $39.33(14)$ \\
\hline $\mathrm{S} 2-\mathrm{O} 7-\mathrm{C} 7-\mathrm{C} 6$ & $-24.5(2)$ & $\mathrm{N} 1-\mathrm{C} 1-\mathrm{C} 2-\mathrm{C} 3$ & $11.3(3)$ \\
\hline $\mathrm{S} 2-\mathrm{O} 7-\mathrm{C} 7-\mathrm{C} 8$ & $157.25(14)$ & $\mathrm{N} 2-\mathrm{S} 2-\mathrm{O} 7-\mathrm{C} 7$ & $40.92(14)$ \\
\hline $\mathrm{S} 2-\mathrm{N} 2-\mathrm{C} 5-\mathrm{Sr} 1^{\mathrm{i}}$ & $167.42(14)$ & $\mathrm{N} 2-\mathrm{S} 2-\mathrm{O} 9-\mathrm{Sr}{ }^{\mathrm{iii}}$ & $31.28(14)$ \\
\hline $\mathrm{S} 2-\mathrm{N} 2-\mathrm{C} 5-\mathrm{O} 6$ & $-171.93(13)$ & $\mathrm{N} 2-\mathrm{C} 5-\mathrm{C} 6-\mathrm{C} 7$ & $9.7(3)$ \\
\hline $\mathrm{S} 2-\mathrm{N} 2-\mathrm{C} 5-\mathrm{C} 6$ & $12.8(2)$ & $\mathrm{C} 1-\mathrm{C} 2-\mathrm{C} 3-\mathrm{O} 3$ & $-7.2(3)$ \\
\hline $\mathrm{O} 2-\mathrm{C} 1-\mathrm{C} 2-\mathrm{C} 3$ & $-162.30(17)$ & $\mathrm{C} 1-\mathrm{C} 2-\mathrm{C} 3-\mathrm{C} 4$ & $167.20(19)$ \\
\hline
\end{tabular}




$\begin{array}{llll}\mathrm{O} 3-\mathrm{S} 1-\mathrm{O} 5-\mathrm{Sr} 1^{\text {iv }} & -74.19(13) & \mathrm{C} 5-\mathrm{C} 6-\mathrm{C} 7-\mathrm{O} 7 & -3.0(3) \\ \mathrm{O} 3-\mathrm{S} 1-\mathrm{N} 1-\mathrm{Sr} 1^{1 \mathrm{ii}} & 116.42(12) & \mathrm{C} 5-\mathrm{C} 6-\mathrm{C} 7-\mathrm{C} 8 & 175.01(18)\end{array}$

Symmetry codes: (i) $-x+2,-y+1,-z+1$; (ii) $-x+1,-y+1,-z+1$; (iii) $x+1, y, z$; (iv) $x-1, y, z$.

Hydrogen-bond geometry $\left(\AA,{ }^{\circ}\right)$

\begin{tabular}{lllll}
\hline$D-\mathrm{H} \cdots A$ & $D-\mathrm{H}$ & $\mathrm{H} \cdots A$ & $D \cdots A$ & $D-\mathrm{H} \cdots A$ \\
\hline $\mathrm{O} 1-\mathrm{H} 1 A \cdots{ }^{\mathrm{v}}$ & $0.79(3)$ & $2.18(3)$ & $2.899(2)$ & $151(3)$ \\
$\mathrm{O} 1-\mathrm{H} 1 B \cdots 8^{\mathrm{vi}}$ & $0.83(3)$ & $2.22(3)$ & $2.9850(19)$ & $152(3)$ \\
$\mathrm{C} 4-\mathrm{H} 4 B \cdots 5^{\mathrm{v}}$ & 0.98 & 2.37 & $3.273(3)$ & 153 \\
$\mathrm{C} 6-\mathrm{H} 6 \cdots 8^{\mathrm{vi}}$ & 0.95 & 2.47 & $3.318(2)$ & 148 \\
\hline
\end{tabular}

Symmetry codes: (v) $x+1 / 2,-y+1 / 2, z-1 / 2$; (vi) $-x+2,-y+1,-z$.

Aquabis $\left(\mu_{3}\right.$-6-methyl-2,2-dioxo-1,2 $\lambda^{6}, 3$-oxathiazin-4-olato)barium(II) (Ba)

\section{Crystal data}

$\left[\mathrm{Ba}\left(\mathrm{C}_{4} \mathrm{H}_{4} \mathrm{NO}_{4} \mathrm{~S}\right)_{2}\left(\mathrm{H}_{2} \mathrm{O}\right)\right]$

$M_{r}=479.64$

Monoclinic, $P 2_{1} / n$

$a=8.2086(5) \AA$

$b=18.8899(11) \AA$

$c=9.8999(6) \AA$

$\beta=99.5197(10)^{\circ}$

$V=1513.93(16) \AA^{3}$

$Z=4$

\section{Data collection}

Bruker PHOTON-100 CMOS diffractometer

Radiation source: sealedtube $\varphi$ and $\omega$ scans

Absorption correction: numerical

(SADABS; Krause et al., 2015)

$T_{\min }=0.309, T_{\max }=0.635$

41654 measured reflections

\section{Refinement}

Refinement on $F^{2}$

Least-squares matrix: full

$R\left[F^{2}>2 \sigma\left(F^{2}\right)\right]=0.032$

$w R\left(F^{2}\right)=0.059$

$S=1.09$

6324 reflections

209 parameters

0 restraints
$F(000)=928$

$D_{\mathrm{x}}=2.104 \mathrm{Mg} \mathrm{m}^{-3}$

Mo $K \alpha$ radiation, $\lambda=0.71073 \AA$

Cell parameters from 9794 reflections

$\theta=3.0-36.3^{\circ}$

$\mu=2.94 \mathrm{~mm}^{-1}$

$T=173 \mathrm{~K}$

Block, colourless

$0.52 \times 0.21 \times 0.20 \mathrm{~mm}$

6324 independent reflections

5218 reflections with $I>2 \sigma(I)$

$R_{\text {int }}=0.048$

$\theta_{\max }=34.3^{\circ}, \theta_{\min }=3.0^{\circ}$

$h=-13 \rightarrow 13$

$k=-29 \rightarrow 29$

$l=-15 \rightarrow 15$

Primary atom site location: isomorphous structure methods

Hydrogen site location: mixed

$\mathrm{H}$ atoms treated by a mixture of independent and constrained refinement

$w=1 /\left[\sigma^{2}\left(F_{\mathrm{o}}^{2}\right)+(0.0158 P)^{2}+2.2204 P\right]$

where $P=\left(F_{\mathrm{o}}^{2}+2 F_{\mathrm{c}}^{2}\right) / 3$

$(\Delta / \sigma)_{\max }=0.001$

$\Delta \rho_{\max }=1.02 \mathrm{e} \AA^{-3}$

$\Delta \rho_{\min }=-0.61$ e $\AA^{-3}$ 


\section{Special details}

Geometry. All esds (except the esd in the dihedral angle between two 1.s. planes) are estimated using the full covariance matrix. The cell esds are taken into account individually in the estimation of esds in distances, angles and torsion angles; correlations between esds in cell parameters are only used when they are defined by crystal symmetry. An approximate (isotropic) treatment of cell esds is used for estimating esds involving l.s. planes.

Fractional atomic coordinates and isotropic or equivalent isotropic displacement parameters $\left(\AA^{2}\right)$

\begin{tabular}{lllll}
\hline & $x$ & $y$ & $z$ & $U_{\mathrm{iso}} * / U_{\mathrm{eq}}$ \\
\hline Ba1 & $0.73689(2)$ & $0.46819(2)$ & $0.43614(2)$ & $0.01376(3)$ \\
S1 & $0.07538(7)$ & $0.32148(3)$ & $0.54490(6)$ & $0.01768(10)$ \\
S2 & $1.36455(6)$ & $0.52303(3)$ & $0.15371(6)$ & $0.01847(11)$ \\
O1 & $0.6927(3)$ & $0.37493(12)$ & $0.2222(2)$ & $0.0313(4)$ \\
H1A & $0.692(5)$ & $0.336(2)$ & $0.213(4)$ & $0.047(12)^{*}$ \\
H1B & $0.664(5)$ & $0.389(2)$ & $0.153(4)$ & $0.041(11)^{*}$ \\
O2 & $0.4531(2)$ & $0.40981(10)$ & $0.48151(19)$ & $0.0255(4)$ \\
O3 & $0.0469(2)$ & $0.28363(10)$ & $0.39659(19)$ & $0.0259(4)$ \\
O4 & $0.1222(3)$ & $0.26718(10)$ & $0.6433(2)$ & $0.0314(4)$ \\
O5 & $-0.0809(2)$ & $0.35404(9)$ & $0.5498(2)$ & $0.0260(4)$ \\
O6 & $1.0152(2)$ & $0.48791(10)$ & $0.33283(18)$ & $0.0227(4)$ \\
O7 & $1.3411(2)$ & $0.44828(10)$ & $0.07381(19)$ & $0.0270(4)$ \\
O8 & $1.3107(2)$ & $0.57651(11)$ & $0.05494(18)$ & $0.0270(4)$ \\
O9 & $1.5363(2)$ & $0.52380(11)$ & $0.21146(18)$ & $0.0257(4)$ \\
N1 & $0.2129(2)$ & $0.37885(10)$ & $0.5425(2)$ & $0.0190(4)$ \\
N2 & $1.2552(2)$ & $0.51888(11)$ & $0.27018(19)$ & $0.0190(4)$ \\
C1 & $0.3361(3)$ & $0.36655(12)$ & $0.4691(2)$ & $0.0185(4)$ \\
C2 & $0.3227(3)$ & $0.30894(13)$ & $0.3700(3)$ & $0.0240(5)$ \\
H2 & 0.416716 & 0.296609 & 0.330515 & $0.029^{*}$ \\
C3 & $0.1826(3)$ & $0.27297(13)$ & $0.3330(3)$ & $0.0233(5)$ \\
C4 & $0.1416(4)$ & $0.22233(17)$ & $0.2179(3)$ & $0.0381(7)$ \\
H4A & 0.104320 & 0.177552 & 0.252407 & $0.057^{*}$ \\
H4B & 0.239932 & 0.213846 & 0.175937 & $0.057^{*}$ \\
H4C & 0.053596 & 0.242128 & 0.149386 & $0.057^{*}$ \\
C5 & $1.1101(3)$ & $0.48350(12)$ & $0.2459(2)$ & $0.0169(4)$ \\
C6 & $1.0711(3)$ & $0.43709(13)$ & $0.1272(2)$ & $0.0213(4)$ \\
H6 & 0.962455 & 0.418511 & 0.104690 & $0.026^{*}$ \\
C7 & $1.1828(3)$ & $0.41992(14)$ & $0.0493(2)$ & $0.0237(5)$ \\
C8 & $1.1637(5)$ & $0.36907(18)$ & $-0.0670(3)$ & $0.0422(8)$ \\
H8A & 1.184568 & 0.393477 & -0.149831 & $0.063^{*}$ \\
H8B & 1.051152 & 0.350013 & -0.082391 & $0.063^{*}$ \\
H8C & 1.242929 & 0.330240 & -0.045421 & $0.063^{*}$ \\
& & & &
\end{tabular}

Atomic displacement parameters $\left(\AA^{2}\right)$

\begin{tabular}{lllllll}
\hline & $U^{11}$ & $U^{22}$ & $U^{33}$ & $U^{12}$ & $U^{13}$ & $U^{23}$ \\
\hline Ba1 & $0.01155(5)$ & $0.01638(6)$ & $0.01386(6)$ & $-0.00196(5)$ & $0.00359(4)$ & $-0.00155(5)$ \\
S1 & $0.0171(2)$ & $0.0136(2)$ & $0.0238(3)$ & $0.00023(19)$ & $0.0076(2)$ & $0.0012(2)$ \\
S2 & $0.0133(2)$ & $0.0298(3)$ & $0.0126(2)$ & $-0.0002(2)$ & $0.00297(18)$ & $0.0006(2)$
\end{tabular}




\begin{tabular}{lllllll} 
O1 & $0.0517(13)$ & $0.0203(10)$ & $0.0211(10)$ & $-0.0050(9)$ & $0.0039(9)$ & $-0.0042(8)$ \\
O2 & $0.0182(8)$ & $0.0281(9)$ & $0.0304(10)$ & $-0.0065(7)$ & $0.0049(7)$ & $0.0006(8)$ \\
O3 & $0.0212(8)$ & $0.0291(9)$ & $0.0286(9)$ & $-0.0067(7)$ & $0.0076(7)$ & $-0.0093(8)$ \\
O4 & $0.0402(11)$ & $0.0195(9)$ & $0.0348(11)$ & $0.0034(8)$ & $0.0072(9)$ & $0.0098(8)$ \\
O5 & $0.0188(8)$ & $0.0224(8)$ & $0.0397(11)$ & $0.0023(7)$ & $0.0135(7)$ & $0.0028(8)$ \\
O6 & $0.0171(7)$ & $0.0319(9)$ & $0.0211(8)$ & $-0.0024(7)$ & $0.0093(6)$ & $-0.0025(7)$ \\
O7 & $0.0230(8)$ & $0.0367(10)$ & $0.0232(9)$ & $0.0001(7)$ & $0.0098(7)$ & $-0.0092(8)$ \\
O8 & $0.0237(8)$ & $0.0377(11)$ & $0.0195(9)$ & $0.0005(8)$ & $0.0026(7)$ & $0.0089(8)$ \\
O9 & $0.0139(7)$ & $0.0438(11)$ & $0.0193(8)$ & $-0.0010(7)$ & $0.0023(6)$ & $0.0032(8)$ \\
N1 & $0.0163(8)$ & $0.0172(9)$ & $0.0244(10)$ & $-0.0023(7)$ & $0.0065(7)$ & $-0.0016(7)$ \\
N2 & $0.0154(8)$ & $0.0290(11)$ & $0.0131(8)$ & $-0.0037(7)$ & $0.0045(7)$ & $-0.0021(7)$ \\
C1 & $0.0146(9)$ & $0.0190(10)$ & $0.0217(11)$ & $0.0016(8)$ & $0.0021(8)$ & $0.0032(8)$ \\
C2 & $0.0227(11)$ & $0.0232(12)$ & $0.0282(13)$ & $0.0035(9)$ & $0.0109(10)$ & $-0.0014(10)$ \\
C3 & $0.0263(12)$ & $0.0225(11)$ & $0.0220(11)$ & $0.0014(9)$ & $0.0072(9)$ & $-0.0030(9)$ \\
C4 & $0.0475(18)$ & $0.0343(15)$ & $0.0342(16)$ & $-0.0044(13)$ & $0.0123(14)$ & $-0.0140(13)$ \\
C5 & $0.0154(9)$ & $0.0202(10)$ & $0.0153(10)$ & $0.0007(8)$ & $0.0028(8)$ & $0.0020(8)$ \\
C6 & $0.0197(10)$ & $0.0264(12)$ & $0.0172(11)$ & $-0.0047(9)$ & $0.0014(8)$ & $-0.0023(9)$ \\
C7 & $0.0283(12)$ & $0.0256(12)$ & $0.0171(11)$ & $-0.0021(10)$ & $0.0036(9)$ & $-0.0017(9)$ \\
C8 & $0.058(2)$ & $0.0421(18)$ & $0.0293(15)$ & $-0.0127(15)$ & $0.0151(14)$ & $-0.0172(13)$ \\
& & & & & & \\
\hline
\end{tabular}

Geometric parameters $\left(\AA,{ }^{\circ}\right)$

\begin{tabular}{llll}
\hline $\mathrm{Ba} 1-\mathrm{Ba} 1^{\mathrm{i}}$ & $4.4491(3)$ & $\mathrm{O} 1-\mathrm{H} 1 \mathrm{~B}$ & $0.74(4)$ \\
$\mathrm{Ba} 1-\mathrm{O} 1$ & $2.732(2)$ & $\mathrm{O} 2-\mathrm{C} 1$ & $1.252(3)$ \\
$\mathrm{Ba} 1-\mathrm{O} 2^{\mathrm{i}}$ & $2.9714(19)$ & $\mathrm{O} 3-\mathrm{C} 3$ & $1.382(3)$ \\
$\mathrm{Ba} 1-\mathrm{O} 2$ & $2.6812(17)$ & $\mathrm{O} 6-\mathrm{C} 5$ & $1.256(3)$ \\
$\mathrm{Ba} 1-\mathrm{O} 5^{\mathrm{ii}}$ & $2.7560(18)$ & $\mathrm{O} 7-\mathrm{C} 7$ & $1.389(3)$ \\
$\mathrm{Ba} 1-\mathrm{O} 6$ & $2.6788(16)$ & $\mathrm{N} 1-\mathrm{C} 1$ & $1.359(3)$ \\
$\mathrm{Ba} 1-\mathrm{O} 6^{\mathrm{iii}}$ & $2.9192(18)$ & $\mathrm{N} 2-\mathrm{C} 5$ & $1.352(3)$ \\
$\mathrm{Ba} 1-\mathrm{O} 9^{\text {ii }}$ & $2.7478(18)$ & $\mathrm{C} 1-\mathrm{C} 2$ & $1.457(3)$ \\
$\mathrm{Ba} 1-\mathrm{N} 1^{\mathrm{i}}$ & $2.921(2)$ & $\mathrm{C} 2-\mathrm{H} 2$ & 0.9500 \\
$\mathrm{Ba} 1-\mathrm{N} 2^{\mathrm{iii}}$ & $2.9077(19)$ & $\mathrm{C} 2-\mathrm{C} 3$ & $1.334(4)$ \\
$\mathrm{Ba} 1-\mathrm{C} 1^{\mathrm{i}}$ & $3.342(2)$ & $\mathrm{C} 3-\mathrm{C} 4$ & $1.483(4)$ \\
$\mathrm{Ba} 1-\mathrm{C} 5^{\mathrm{iii}}$ & $3.318(2)$ & $\mathrm{C} 4-\mathrm{H} 4 \mathrm{~A}$ & 0.9800 \\
$\mathrm{~S} 1-\mathrm{O} 3$ & $1.6149(19)$ & $\mathrm{C} 4-\mathrm{H} 4 \mathrm{~B}$ & 0.9800 \\
$\mathrm{~S} 1-\mathrm{O} 4$ & $1.4232(19)$ & $\mathrm{C} 4-\mathrm{H} 4 \mathrm{C}$ & 0.9800 \\
$\mathrm{~S} 1-\mathrm{O} 5$ & $1.4309(17)$ & $\mathrm{C} 5-\mathrm{C} 6$ & $1.459(3)$ \\
$\mathrm{S} 1-\mathrm{N} 1$ & $1.568(2)$ & $\mathrm{C} 6-\mathrm{H} 6$ & 0.9500 \\
$\mathrm{~S} 2-\mathrm{O} 7$ & $1.6143(19)$ & $\mathrm{C} 6-\mathrm{C} 7$ & $1.332(3)$ \\
$\mathrm{S} 2-\mathrm{O} 8$ & $1.4245(19)$ & $\mathrm{C} 7-\mathrm{C} 8$ & $1.488(4)$ \\
$\mathrm{S} 2-\mathrm{O} 9$ & $1.4313(17)$ & $\mathrm{C} 8-\mathrm{H} 8 \mathrm{~A}$ & 0.9800 \\
$\mathrm{~S} 2-\mathrm{N} 2$ & $1.5758(19)$ & $\mathrm{C} 8-\mathrm{H} 8 \mathrm{~B}$ & 0.9800 \\
$\mathrm{O} 1-\mathrm{H} 1 \mathrm{~A}$ & $0.75(4)$ & $\mathrm{C} 8-\mathrm{H} 8 \mathrm{C}$ & 0.9800 \\
& & & $115.39(12)$ \\
$\mathrm{O} 1-\mathrm{Ba} 1-\mathrm{Ba}^{\mathrm{i}}$ & $112.59(5)$ & $\mathrm{O} 4-\mathrm{S} 1-\mathrm{O} 5$ & $113.25(12)$ \\
$\mathrm{O} 1-\mathrm{Ba} 1-\mathrm{O}^{\mathrm{i}}$ & $134.76(6)$ & $\mathrm{O} 4-\mathrm{S} 1-\mathrm{N} 1$ & $103.03(11)$ \\
$\mathrm{O} 1-\mathrm{Ba} 1-\mathrm{O} 5^{\mathrm{ii}}$ & $78.79(7)$ & $\mathrm{O} 5-\mathrm{S} 1-\mathrm{O} 3$ & $110.81(11)$ \\
$\mathrm{O} 1-\mathrm{Ba} 1-\mathrm{O}^{\mathrm{iii}}$ & $140.94(6)$ & $\mathrm{O} 5-\mathrm{S} 1-\mathrm{N} 1$ &
\end{tabular}




\begin{tabular}{|c|c|c|c|}
\hline $\mathrm{O} 1-\mathrm{Ba} 1-\mathrm{O}^{\text {iv }}$ & $68.53(7)$ & $\mathrm{N} 1-\mathrm{S} 1-\mathrm{O} 3$ & $106.77(10)$ \\
\hline $\mathrm{O} 1-\mathrm{Ba} 1-\mathrm{N} 1^{\mathrm{i}}$ & $133.85(6)$ & $\mathrm{O} 8-\mathrm{S} 2-\mathrm{O} 7$ & $106.60(11)$ \\
\hline $\mathrm{O} 1-\mathrm{Ba} 1-\mathrm{N} 2^{\mathrm{iii}}$ & $143.83(6)$ & $\mathrm{O} 8-\mathrm{S} 2-\mathrm{O} 9$ & $116.14(11)$ \\
\hline $\mathrm{O} 1-\mathrm{Ba} 1-\mathrm{C}^{\mathrm{i}}$ & $144.37(7)$ & $\mathrm{O} 8-\mathrm{S} 2-\mathrm{N} 2$ & $112.82(11)$ \\
\hline $\mathrm{O} 1-\mathrm{Ba} 1-\mathrm{C} 5^{\mathrm{iii}}$ & $153.32(7)$ & $\mathrm{O} 9-\mathrm{S} 2-\mathrm{O} 7$ & $103.55(11)$ \\
\hline $\mathrm{O} 2^{\mathrm{i}}-\mathrm{Ba} 1-\mathrm{Ba} 1^{\mathrm{i}}$ & $35.84(3)$ & $\mathrm{O} 9-\mathrm{S} 2-\mathrm{N} 2$ & $110.54(10)$ \\
\hline $\mathrm{O} 2-\mathrm{Ba} 1-\mathrm{Ba}^{\mathrm{i}}$ & $40.45(4)$ & $\mathrm{N} 2-\mathrm{S} 2-\mathrm{O} 7$ & $106.20(10)$ \\
\hline $\mathrm{O} 2-\mathrm{Ba} 1-\mathrm{O} 1$ & $81.78(6)$ & $\mathrm{Ba} 1-\mathrm{O} 1-\mathrm{H} 1 \mathrm{~A}$ & $137(3)$ \\
\hline $\mathrm{O} 2-\mathrm{Ba} 1-\mathrm{O} 2^{\mathrm{i}}$ & $76.29(6)$ & $\mathrm{Ba} 1-\mathrm{O} 1-\mathrm{H} 1 \mathrm{~B}$ & $118(3)$ \\
\hline $\mathrm{O} 2-\mathrm{Ba} 1-\mathrm{O} 5^{\mathrm{ii}}$ & $92.16(5)$ & $\mathrm{H} 1 \mathrm{~A}-\mathrm{O} 1-\mathrm{H} 1 \mathrm{~B}$ & $105(4)$ \\
\hline $\mathrm{O} 2-\mathrm{Ba} 1-\mathrm{O}^{\mathrm{iii}}$ & $119.87(5)$ & $\mathrm{Ba} 1-\mathrm{O} 2-\mathrm{Ba}^{\mathrm{i}}$ & $103.71(6)$ \\
\hline $\mathrm{O} 2-\mathrm{Ba} 1-\mathrm{O}^{\mathrm{iv}}$ & $82.65(5)$ & $\mathrm{C} 1-\mathrm{O} 2-\mathrm{Ba} 1$ & $157.81(16)$ \\
\hline $\mathrm{O} 2-\mathrm{Ba} 1-\mathrm{N} 1^{\mathrm{i}}$ & $120.71(5)$ & $\mathrm{C} 1-\mathrm{O} 2-\mathrm{Ba}^{\mathrm{i}}$ & $95.98(14)$ \\
\hline $\mathrm{O} 2-\mathrm{Ba} 1-\mathrm{N} 2^{\mathrm{iii}}$ & $75.13(5)$ & $\mathrm{C} 3-\mathrm{O} 3-\mathrm{S} 1$ & $118.19(16)$ \\
\hline $\mathrm{O} 2^{\mathrm{i}}-\mathrm{Ba} 1-\mathrm{C}^{\mathrm{i}}$ & $21.87(5)$ & $\mathrm{S} 1-\mathrm{O} 5-\mathrm{Ba} 1^{\mathrm{iv}}$ & $138.72(10)$ \\
\hline $\mathrm{O} 2-\mathrm{Ba} 1-\mathrm{C} 1^{\mathrm{i}}$ & $97.82(6)$ & $\mathrm{Ba} 1-\mathrm{O} 6-\mathrm{Ba} 1^{\mathrm{iii}}$ & $105.57(5)$ \\
\hline $\mathrm{O} 2-\mathrm{Ba} 1-\mathrm{C} 5^{\mathrm{iii}}$ & $99.04(5)$ & $\mathrm{C} 5-\mathrm{O} 6-\mathrm{Ba} 1^{\mathrm{iii}}$ & $97.13(14)$ \\
\hline $\mathrm{O} 2^{\mathrm{i}}-\mathrm{Ba} 1-\mathrm{C} 5^{\mathrm{iii}}$ & $70.11(5)$ & $\mathrm{C} 5-\mathrm{O} 6-\mathrm{Ba} 1$ & $156.59(16)$ \\
\hline 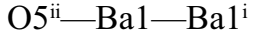 & $122.42(4)$ & $\mathrm{C} 7-\mathrm{O} 7-\mathrm{S} 2$ & $117.07(15)$ \\
\hline $\mathrm{O} 5^{\mathrm{ii}}-\mathrm{Ba} 1-\mathrm{O} 2^{\mathrm{i}}$ & $140.25(5)$ & $\mathrm{S} 2-\mathrm{O} 9-\mathrm{Ba} 1^{\mathrm{ii}}$ & $137.59(10)$ \\
\hline $\mathrm{O} 5^{\mathrm{ii}}-\mathrm{Ba} 1-\mathrm{O}^{\mathrm{iii}}$ & $68.99(5)$ & $\mathrm{S} 1-\mathrm{N} 1-\mathrm{Ba} 1^{\mathrm{i}}$ & $140.88(10)$ \\
\hline $\mathrm{O} 5^{\mathrm{ii}}-\mathrm{Ba} 1-\mathrm{N} 1^{\mathrm{i}}$ & $133.14(6)$ & $\mathrm{C} 1-\mathrm{N} 1-\mathrm{Ba} 1^{\mathrm{i}}$ & $95.70(13)$ \\
\hline $\mathrm{O} 5^{\mathrm{ii}}-\mathrm{Ba} 1-\mathrm{N} 2^{\mathrm{iii}}$ & $74.73(6)$ & $\mathrm{C} 1-\mathrm{N} 1-\mathrm{S} 1$ & $119.41(17)$ \\
\hline $\mathrm{O} 5^{\mathrm{ii}}-\mathrm{Ba} 1-\mathrm{C}^{\mathrm{i}}$ & $136.60(6)$ & $\mathrm{S} 2-\mathrm{N} 2-\mathrm{Ba} 1^{\mathrm{iii}}$ & $143.73(10)$ \\
\hline $\mathrm{O} 5^{\mathrm{ii}}-\mathrm{Ba} 1-\mathrm{C} 5^{\mathrm{iii}}$ & $74.53(6)$ & $\mathrm{C} 5-\mathrm{N} 2-\mathrm{Ba} 1^{\mathrm{iii}}$ & $95.30(13)$ \\
\hline $\mathrm{O} 6-\mathrm{Ba} 1-\mathrm{Ba}^{\mathrm{i}}$ & $155.73(4)$ & $\mathrm{C} 5-\mathrm{N} 2-\mathrm{S} 2$ & 119.09 (16) \\
\hline $\mathrm{O}^{\mathrm{iii}}-\mathrm{Ba} 1-\mathrm{Ba}^{\mathrm{i}}$ & $103.27(3)$ & $\mathrm{O} 2-\mathrm{C} 1-\mathrm{Ba}^{\mathrm{i}}$ & $62.15(13)$ \\
\hline $\mathrm{O} 6-\mathrm{Ba} 1-\mathrm{O} 1$ & $78.82(6)$ & $\mathrm{O} 2-\mathrm{C} 1-\mathrm{N} 1$ & $117.5(2)$ \\
\hline $\mathrm{O}^{\mathrm{iii}}-\mathrm{Ba} 1-\mathrm{O} 2^{\mathrm{i}}$ & $83.93(5)$ & $\mathrm{O} 2-\mathrm{C} 1-\mathrm{C} 2$ & $121.7(2)$ \\
\hline $\mathrm{O} 6-\mathrm{Ba} 1-\mathrm{O} 2$ & $160.13(6)$ & $\mathrm{N} 1-\mathrm{C} 1-\mathrm{Ba}^{\mathrm{i}}$ & $60.43(12)$ \\
\hline $\mathrm{O} 6-\mathrm{Ba} 1-\mathrm{O} 2^{\mathrm{i}}$ & $121.09(5)$ & $\mathrm{N} 1-\mathrm{C} 1-\mathrm{C} 2$ & $120.5(2)$ \\
\hline $\mathrm{O} 6-\mathrm{Ba} 1-\mathrm{O}^{\mathrm{ii}}$ & $79.94(5)$ & $\mathrm{C} 2-\mathrm{C} 1-\mathrm{Ba}^{\mathrm{i}}$ & $152.82(16)$ \\
\hline $\mathrm{O} 6-\mathrm{Ba} 1-\mathrm{O}^{\mathrm{iii}}$ & $74.43(5)$ & $\mathrm{C} 1-\mathrm{C} 2-\mathrm{H} 2$ & 119.0 \\
\hline $\mathrm{O} 6-\mathrm{Ba} 1-\mathrm{O}^{\mathrm{iv}}$ & $94.15(5)$ & $\mathrm{C} 3-\mathrm{C} 2-\mathrm{C} 1$ & $122.1(2)$ \\
\hline $\mathrm{O} 6^{\mathrm{iii}}-\mathrm{Ba} 1-\mathrm{N} 1^{\mathrm{i}}$ & $65.83(5)$ & $\mathrm{C} 3-\mathrm{C} 2-\mathrm{H} 2$ & 119.0 \\
\hline $\mathrm{O} 6-\mathrm{Ba} 1-\mathrm{N} 1^{\mathrm{i}}$ & $76.77(5)$ & $\mathrm{O} 3-\mathrm{C} 3-\mathrm{C} 4$ & $110.3(2)$ \\
\hline $\mathrm{O} 6-\mathrm{Ba} 1-\mathrm{N} 2^{\mathrm{iii}}$ & $119.39(5)$ & $\mathrm{C} 2-\mathrm{C} 3-\mathrm{O} 3$ & $121.6(2)$ \\
\hline $\mathrm{O}^{\mathrm{iii}}-\mathrm{Ba} 1-\mathrm{Cl}^{\mathrm{i}}$ & $69.44(5)$ & $\mathrm{C} 2-\mathrm{C} 3-\mathrm{C} 4$ & $128.0(2)$ \\
\hline $\mathrm{O} 6-\mathrm{Ba} 1-\mathrm{C}^{\mathrm{i}}$ & $100.43(5)$ & $\mathrm{C} 3-\mathrm{C} 4-\mathrm{H} 4 \mathrm{~A}$ & 109.5 \\
\hline $\mathrm{O} 6-\mathrm{Ba} 1-\mathrm{C}^{5 \mathrm{iii}}$ & $96.38(5)$ & $\mathrm{C} 3-\mathrm{C} 4-\mathrm{H} 4 \mathrm{~B}$ & 109.5 \\
\hline $\mathrm{O}^{\mathrm{iii}}-\mathrm{Ba} 1-\mathrm{C} 5^{\mathrm{iii}}$ & $22.06(5)$ & $\mathrm{C} 3-\mathrm{C} 4-\mathrm{H} 4 \mathrm{C}$ & 109.5 \\
\hline $\mathrm{O} 9^{\mathrm{iv}}-\mathrm{Ba} 1-\mathrm{Ba} 1^{\mathrm{i}}$ & $72.06(4)$ & $\mathrm{H} 4 \mathrm{~A}-\mathrm{C} 4-\mathrm{H} 4 \mathrm{~B}$ & 109.5 \\
\hline $\mathrm{O} 9^{\mathrm{iv}}-\mathrm{Ba} 1-\mathrm{O} 2^{\mathrm{i}}$ & $69.77(5)$ & $\mathrm{H} 4 \mathrm{~A}-\mathrm{C} 4-\mathrm{H} 4 \mathrm{C}$ & 109.5 \\
\hline $\mathrm{O}^{\mathrm{iv}}-\mathrm{Ba} 1-\mathrm{O}^{\mathrm{ii}}$ & $147.31(6)$ & $\mathrm{H} 4 \mathrm{~B}-\mathrm{C} 4-\mathrm{H} 4 \mathrm{C}$ & 109.5 \\
\hline $\mathrm{O} 9^{\mathrm{iv}}-\mathrm{Ba} 1-\mathrm{O}^{\mathrm{iii}}$ & $140.57(6)$ & $\mathrm{O} 6-\mathrm{C} 5-\mathrm{Ba} 1^{\mathrm{iii}}$ & $60.81(12)$ \\
\hline $\mathrm{O} 9^{\mathrm{iv}}-\mathrm{Ba} 1-\mathrm{N} 1^{\mathrm{i}}$ & $74.86(6)$ & $\mathrm{O} 6-\mathrm{C} 5-\mathrm{N} 2$ & $118.3(2)$ \\
\hline $\mathrm{O} 9^{\mathrm{iv}}-\mathrm{Ba} 1-\mathrm{N} 2^{\mathrm{iii}}$ & $133.52(6)$ & $\mathrm{O} 6-\mathrm{C} 5-\mathrm{C} 6$ & $120.9(2)$ \\
\hline $\mathrm{O} 9^{\mathrm{iv}}-\mathrm{Ba} 1-\mathrm{C} 1^{\mathrm{i}}$ & $76.05(6)$ & $\mathrm{N} 2-\mathrm{C} 5-\mathrm{Ba} 1^{\mathrm{iii}}$ & $60.77(11)$ \\
\hline
\end{tabular}




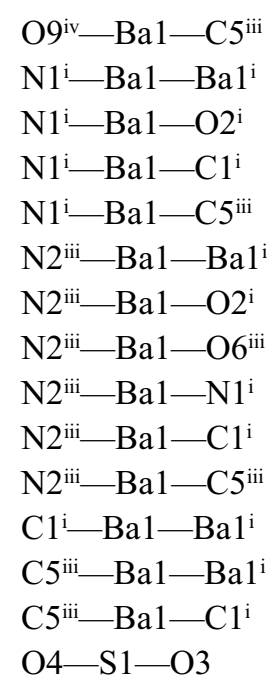

$138.14(6)$

80.31 (4)

$44.54(5)$

$23.87(5)$

$68.44(6)$

$64.48(4)$

$65.57(5)$

$45.20(5)$

$82.24(6)$

$67.43(6)$

$23.94(5)$

57.47 (4)

$82.39(4)$

$62.23(6)$

$106.60(11)$

$-152.6(4)$

$-177.7(3)$

$-25.2(2)$

-3.7 (6)

148.9 (2)

$-165.9(4)$

$-20.6(2)$

$173.5(3)$

$-11.1(5)$

$154.73(19)$

$25.6(2)$

$-148.52(19)$

20.6 (2)

-154.73 (19)

$-74.1(4)$

$-77.8(5)$

$-18.7(3)$

$165.55(19)$

$162.0(2)$

$-172.35(18)$

13.5 (3)

$-24.1(3)$

157.7 (2)

$168.0(2)$

$-171.40(18)$

$13.3(3)$

$-164.1(2)$

$-73.61(18)$

$117.32(17)$
$\mathrm{N} 2-\mathrm{C} 5-\mathrm{C} 6$

$\mathrm{C} 6-\mathrm{C} 5-\mathrm{Ba} 1^{\mathrm{iii}}$

$\mathrm{C} 5-\mathrm{C} 6-\mathrm{H} 6$

$\mathrm{C} 7-\mathrm{C} 6-\mathrm{C} 5$

$\mathrm{C} 7-\mathrm{C} 6-\mathrm{H} 6$

$\mathrm{O} 7-\mathrm{C} 7-\mathrm{C} 8$

$\mathrm{C} 6-\mathrm{C} 7-\mathrm{O} 7$

$\mathrm{C} 6-\mathrm{C} 7-\mathrm{C} 8$

C7-C $8-\mathrm{H} 8 \mathrm{~A}$

C7-C $8-\mathrm{H} 8 \mathrm{~B}$

$\mathrm{C} 7-\mathrm{C} 8-\mathrm{H} 8 \mathrm{C}$

$\mathrm{H} 8 \mathrm{~A}-\mathrm{C} 8-\mathrm{H} 8 \mathrm{~B}$

$\mathrm{H} 8 \mathrm{~A}-\mathrm{C} 8-\mathrm{H} 8 \mathrm{C}$

$\mathrm{H} 8 \mathrm{~B}-\mathrm{C} 8-\mathrm{H} 8 \mathrm{C}$

$\mathrm{O} 3-\mathrm{S} 1-\mathrm{N} 1-\mathrm{C} 1$

$\mathrm{O} 4-\mathrm{S} 1-\mathrm{O} 3-\mathrm{C} 3$

$\mathrm{O} 4-\mathrm{S} 1-\mathrm{O} 5-\mathrm{Ba}^{\text {iv }}$

$\mathrm{O} 4-\mathrm{S} 1-\mathrm{N} 1-\mathrm{Ba} 1^{\mathrm{i}}$

$\mathrm{O} 4-\mathrm{S} 1-\mathrm{N} 1-\mathrm{C} 1$

$\mathrm{O} 5-\mathrm{S} 1-\mathrm{O} 3-\mathrm{C} 3$

$\mathrm{O} 5-\mathrm{S} 1-\mathrm{N} 1-\mathrm{Ba}^{\mathrm{i}}$

$\mathrm{O} 5-\mathrm{S} 1-\mathrm{N} 1-\mathrm{C} 1$

$\mathrm{O} 6-\mathrm{C} 5-\mathrm{C} 6-\mathrm{C} 7$

$\mathrm{O} 7-\mathrm{S} 2-\mathrm{O} 9-\mathrm{Ba}^{1 \mathrm{ii}}$

O7-S2-N2-Ba $1^{\text {iii }}$

$\mathrm{O} 7-\mathrm{S} 2-\mathrm{N} 2-\mathrm{C} 5$

$\mathrm{O} 8-\mathrm{S} 2-\mathrm{O} 7-\mathrm{C} 7$

$\mathrm{O} 8-\mathrm{S} 2-\mathrm{O} 9-\mathrm{Ba} 1^{\mathrm{ii}}$

$\mathrm{O} 8-\mathrm{S} 2-\mathrm{N} 2-\mathrm{Ba}^{1 \mathrm{ii}}$

$\mathrm{O} 8-\mathrm{S} 2-\mathrm{N} 2-\mathrm{C} 5$

$\mathrm{O} 9-\mathrm{S} 2-\mathrm{O} 7-\mathrm{C} 7$

O9- $2-\mathrm{N} 2-\mathrm{Ba}^{\mathrm{iii}}$

$\mathrm{O} 9-\mathrm{S} 2-\mathrm{N} 2-\mathrm{C} 5$

$\mathrm{N} 1-\mathrm{S} 1-\mathrm{O} 3-\mathrm{C} 3$

$\mathrm{N} 1-\mathrm{S} 1-\mathrm{O} 5-\mathrm{Ba} 1^{\text {iv }}$

$\mathrm{N} 1-\mathrm{C} 1-\mathrm{C} 2-\mathrm{C} 3$

N2-S2-O7-C7

$\mathrm{N} 2-\mathrm{S} 2-\mathrm{O} 9-\mathrm{Ba} 1^{\mathrm{ii}}$

N2- C5-C6-C7

$\mathrm{C} 1-\mathrm{C} 2-\mathrm{C} 3-\mathrm{O} 3$

$\mathrm{C} 1-\mathrm{C} 2-\mathrm{C} 3-\mathrm{C} 4$

$\mathrm{C} 5-\mathrm{C} 6-\mathrm{C} 7-\mathrm{O} 7$

$\mathrm{C} 5-\mathrm{C} 6-\mathrm{C} 7-\mathrm{C} 8$
$120.65(19)$

$158.10(16)$

118.9

122.1 (2)

118.9

111.1 (2)

$121.2(2)$

$127.6(3)$

109.5

109.5

109.5

109.5

109.5

109.5

$-33.6(2)$

-84.9 (2)

$170.65(15)$

$-125.67(17)$

$83.4(2)$

$153.20(18)$

$5.8(2)$

$-145.06(19)$

$-165.8(2)$

$-80.89(17)$

$124.24(18)$

-35.3 (2)

-79.77 (19)

162.65 (14)

$-119.34(18)$

81.1 (2)

157.23 (18)

$12.6(2)$

-146.95 (19)

36.4 (2)

40.3 (2)

9.8 (4)

40.8 (2)

32.5 (2)

9.4 (4)

$-6.5(4)$

$168.5(3)$

-3.1 (4)

174.7 (3)

Symmetry codes: (i) $-x+1,-y+1,-z+1$; (ii) $x+1, y, z$; (iii) $-x+2,-y+1,-z+1$; (iv) $x-1, y, z$. 
supporting information

Hydrogen-bond geometry $\left(\AA,{ }^{\circ}\right)$

\begin{tabular}{lllll}
\hline$D-\mathrm{H} \cdots A$ & $D-\mathrm{H}$ & $\mathrm{H} \cdots A$ & $D \cdots A$ & $D-\mathrm{H} \cdots A$ \\
\hline $\mathrm{O} 1-\mathrm{H} 1 A \cdots \mathrm{O} 4^{\mathrm{v}}$ & $0.74(4)$ & $2.12(4)$ & $2.829(3)$ & $162(4)$ \\
$\mathrm{O} 1-\mathrm{H} 1 B \cdots 8^{\text {vi }}$ & $0.74(4)$ & $2.20(4)$ & $2.889(3)$ & $156(4)$ \\
$\mathrm{C} 4-\mathrm{H} 4 B \cdots 5^{\mathrm{v}}$ & 0.98 & 2.44 & $3.361(4)$ & 155 \\
$\mathrm{C} 6-\mathrm{H} 6 \cdots 8^{\mathrm{vi}}$ & 0.95 & 2.52 & $3.357(3)$ & 147
\end{tabular}

Symmetry codes: (v) $x+1 / 2,-y+1 / 2, z-1 / 2$; (vi) $-x+2,-y+1,-z$. 